\title{
Review Article \\ Geometrical and P.D.E. Methods in the Treatment of the Theory of Shells: Comparing Euclidean and Affine Approaches
}

\author{
Salvador Gigena, ${ }^{1,2}$ Daniel Abud, ${ }^{3}$ and Moisés Binia ${ }^{2,3}$ \\ ${ }^{1}$ Departamento de Matemáticas, Facultad de Ciencias Exactas, Ingeniería y Agrimensura, Universidad Nacional de Rosario, \\ Avenida Pellegrini 250, 2000 Rosario, Argentina \\ ${ }^{2}$ Departamento de Matemáticas, Facultad de Ciencias Exactas, Físicas y Naturales, Universidad Nacional de Córdoba, \\ Avenida Velez Sarsfield 1611, 5000 Córdoba, Argentina \\ ${ }^{3}$ Departamento de Física, Facultad de Ciencias Exactas, Físicas y Naturales, Universidad Nacional de Córdoba, \\ Avenida Velez Sarsfield 1611, 5000 Córdoba, Argentina
}

Correspondence should be addressed to Salvador Gigena; sgigena@fceia.unr.edu.ar

Received 30 June 2013; Accepted 27 November 2013; Published 23 February 2014

Academic Editor: Bennett Palmer

Copyright (c) 2014 Salvador Gigena et al. This is an open access article distributed under the Creative Commons Attribution License, which permits unrestricted use, distribution, and reproduction in any medium, provided the original work is properly cited.

\begin{abstract}
The use of differential equations methods in the approach, treatment, and solution of problems in diverse areas of geometry, particularly in affine differential geometry is well known and prolific, where they have proven to be quite fruitful when it comes to the obtainment of definite results. It is perhaps lesser known that the same kind of those very same methods has been and is currently being used to treat developments in some specific areas of applied sciences, such as the theory of shells where, similarly, they can be proven to be quite effective as well. In this paper we precisely show that such is the case in two particular, related instances: the historic approach of the classical, Euclidean part of the theory pursued by Fritz John, in the past century, and the more recent expositions that we ourselves have dedicated to the affine counterpart of the theory.
\end{abstract}

\section{Introduction}

The use of differential equations methods in the approach, treatment, and solution of problems in diverse aspects of geometry, particularly in affine differential geometry, is quite well known and efficient. See, for instance, [1-21] and further references therein. On the other hand, it is not quite well known that the applications of geometrical combined with P.D.E. methods may be quite efficient as well in the consideration and treatment of a very specific topic of applied mathematics, namely, the theory of shells. In fact, most of the people working in the area of engineering are not aware of that particular approach initiated in the past century by John $[22,23]$, for the usual Euclidean case, that is, invariant under the action of the Euclidean group. On our hand, inspired by that very same source, we have developed in recent years an alternative approach to the theory which is, in this new situation and context, invariant under the action of the unimodular affine group. See $[24,25]$ and further references therein.
In fact the usual, general theory of thin shells has been historically developed in a great variety of ways and accessed by different authors based, from the geometrical point of view, on the classical theory of surfaces in three-dimensional space, particularly with respect to the invariants of the Euclidean group, $\operatorname{ASO}(3, \mathrm{R})$, that is, the group of transformations generated by translations and rotations of the space [22, 23, 26-39]. Hence, what is called "normal" is the Euclidean one, and the "distance" is measured with respect to the norm induced by the usual, positive definite scalar product of vectors, which is the main, fundamental invariant in Euclidean geometry. Moreover, it should also be emphasized that its realm deals with a topic of mathematics with a rich history and many, diverse applications to the real world: engineering, industry, avionics, and so on.

On our part, we have been working more recently on an alternative foundation and development of the theory of shells which is invariant under the action of the unimodular affine group, $\operatorname{ASL}(3, \mathbb{R})$. Thus, for the case in treatment, this gives rise to the so-called affine geometry of surfaces. For a 
given surface in the three-dimensional space we use, within this context, concepts such as "affine normal" and "affine distance," corresponding to the above mentioned ones in Euclidean geometry. See our previous articles [24, 25], for full details.

In this, mainly intended, survey paper we make an exposition of that alternative foundation and development of the theory. In brief summary, the contents presented here are the following. We start by introducing, in Section 2, an abbreviated version of the concepts of Euclidean and affine shells, previously treated in the latter cited articles. The exposition of compatibility conditions occupies Section 3, already showing from the beginning that the mainstream of the subject lies precisely in geometry and partial differential equations, since those very important conditions derive from the very well-known concept of integrability conditions. The basic inequalities of the theory, a topic inspired precisely in P.D.E. methods, are presented in Section 4. The further development of the theory requires the strain-stress relations in affine shells, with a combination and intercourse of geometrical and physical concepts, which are treated in Section 5. Once again, the use of P.D.E. methods appears with the estimates for the $L_{2}$-norms of second order derivatives, which occupy Section 6, while the corresponding estimates of higher order derivatives take place in Section 7. Finally, Section 8 is dedicated to presenting the recovery of the deformed middle surface, which is obtained through the contribution of the fundamental theorem of unimodular affine surfaces.

\section{Euclidean and Affine Shells}

We consider the middle surface of a (solid) shell in its original (undeformed) state, denoted by $M_{0}$, parametrized locally by a vector function $X_{0}: U \rightarrow \mathbb{R}^{3}$, where $U \subset \mathbb{R}^{2}$, which is assumed to be smooth enough. Coordinates in the domain are denoted by $\left(u^{1}, u^{2}\right)$. Thus, we can write locally $M_{0}=$ $X_{0}(U)$ and assume besides, as it is usually done, that $X_{0}$ is topological immersion (embedding).

Particles in the original state have curvilinear Lagrange coordinates $\left(U^{1}, U^{2}, U^{3}\right)$ that for our present purposes shall be chosen in a special way: $\left(U^{1}, U^{2}, U^{3}\right)=\left(u^{1}, u^{2}, u\right)$ if we represent them by equation $X\left(u^{1}, u^{2}, u\right)=X_{u}\left(u^{1}, u^{2}\right)=$ $X_{0}\left(u^{1}, u^{2}\right)+u \overrightarrow{\mathbf{n}}$, where we have obviously extended the previous function to $X: U \times(-h, h) \rightarrow \mathbb{R}^{3}$, and $\overrightarrow{\mathbf{n}}$ is the vector field normal to the middle surface. This normal can be the Euclidean normal, $N_{e u}$, of the classical, Euclidean theory of surfaces or the unimodular affine normal, $N_{u a}$, of our own, current development. In each case we shall clarify the situation when we deal with one or the other.

In the Euclidean case we shall use the following notations regarding the main geometrical objects, defined on the middle surface prior to deformation, which take part in the formulation of the theory [22-25]:

$$
I_{e u}=\sum_{\alpha, \beta} a_{\alpha \beta} d u^{\alpha} d u^{\beta} \quad \text { with } a_{\alpha \beta}=\frac{\partial X_{0}}{\partial u^{\alpha}} \cdot \frac{\partial X_{0}}{\partial u^{\beta}}
$$

denotes the Euclidean first fundamental form, while with the expression

$$
I I_{e u}=\sum_{\alpha, \beta} L_{\alpha \beta} d u^{\alpha} d u^{\beta} \quad \text { where } L_{\alpha \beta}=N_{e u} \cdot \frac{\partial^{2} X_{0}}{\partial u^{\beta} \partial u^{\alpha}}
$$

we represent the second fundamental form and with

$$
\begin{aligned}
& I I I_{e u}=\sum_{\alpha, \beta} M_{\alpha \beta} d u^{\alpha} d u^{\beta}, \\
& \text { where } M_{\alpha \beta}=\sum_{\lambda} L_{\alpha \lambda} L_{\beta}^{\lambda}=\sum_{\gamma \lambda} a^{\gamma \lambda} L_{\alpha \lambda} L_{\beta \gamma},
\end{aligned}
$$

the Euclidean third fundamental form.

In the state previous to deformation the border of the shell is made up of two "faces," which are surfaces parallel to the middle surface $M_{0}$ at respective distance $h$, measured along the Euclidean normal $N_{e u}$, and of the "border" constituted by segments normal to the faces. Therefore, along the normal to $M_{0}$ coordinates $U^{1}$ and $U^{2}$ remain constant while $U^{3}:=u$ measures the signed distance from $M_{0}$. Faces can be represented, then, by equations $U^{3}=u= \pm h$ while the middle surface is given by $U^{3}=u=0$.

Now if $a_{\alpha \beta}, L_{\alpha \beta}$, and $M_{\alpha \beta}$ are, respectively, the coefficients of the first, second, and third Euclidean fundamental forms of the middle surface $M_{0}$, the Euclidean structure of the ambient space induces a Riemannian structure on the shell and we can obtain, by means of straightforward computations, the following expressions in the above normal coordinates $\left(U^{1}, U^{2}, U^{3}\right)=\left(U^{1}, U^{2}, u\right)[22,23]$ :

$$
\begin{gathered}
A_{\alpha \beta}=\frac{\partial X}{\partial u^{\alpha}} \cdot \frac{\partial X}{\partial u^{\beta}}=a_{\alpha \beta}-2 u L_{\alpha \beta}+u^{2} M_{\alpha \beta}, \\
A_{\alpha 3}=A_{3 \alpha}=\frac{\partial X}{\partial u^{\alpha}} \cdot \frac{\partial X}{\partial u}=\frac{\partial X}{\partial u^{\alpha}} \cdot N_{e u}=0, \\
A_{33}=\frac{\partial X}{\partial u} \cdot \frac{\partial X}{\partial u}=N_{e u} \cdot N_{e u}=1 .
\end{gathered}
$$

Corresponding to the shell and its middle surface, in the state previous to deformation, we can consider the geometrical objects belonging to the shell in the deformed state that we shall denote with an upper right asterisk. Thus, for example, $X_{0}^{*}: U \rightarrow \mathbb{R}^{3}$, where $U \subset \mathbb{R}^{2}$, represents the parametrization of the deformed middle surface $M_{0}^{*}=$ $X_{0}^{*}(U)$, and we remark that the domain of definition of this immersion, $U \subset \mathbb{R}^{2}$, and the parameters $\left(u^{1}, u^{2}\right)$ used in it are the same as those belonging to the middle surface of the shell in the original state, previous to deformation.

Consequently, the rest of geometrical objects change from one state to the other and the problem is to determine the nature and extension of such changes for every one of them reducing, under appropriate hypotheses, the obtainable information to both middle surfaces. One such hypothesis is the one concerning the comparison of the thickness parameter $h$, which is usually assumed to be small with respect to the other dimensions of the shell. This introduces in the theory the concept of "thin" shell which has important uses and applications. 
In order to consider next the unimodular affine geometry of surfaces, we assume a defined, in the ambient space $\mathbb{R}^{3}$, exterior 3-form or nontrivial determinant function, denoted by the symbol $[,]=$, det. Then, given the same previous mean surface, we can represent the objects of that geometry by the following expressions.

First, for the construction of the unimodular first fundamental form we define

$$
h_{\alpha \beta}:=\left[\frac{\partial X_{0}}{\partial u^{1}}, \frac{\partial X_{0}}{\partial u^{2}}, \frac{\partial^{2} X_{0}}{\partial u^{\alpha} \partial u^{\beta}}\right] .
$$

Then, by assuming that the surface is nondegenerate, that is, $H=\operatorname{det}\left(h_{\alpha \beta}\right) \neq 0$, we can write $g_{\alpha \beta}=|H|^{-1 / 4} h_{\alpha \beta}$, obtaining the unimodular affine first fundamental form expressed by equation

$$
I_{u a}=\sum_{\alpha, \beta} g_{\alpha \beta} d u^{\alpha} d u^{\beta} .
$$

It follows that the latter turns out to be a semi-Riemannian structure, which we shall also refer to as pseudometric. See $[2-4,7,8,11,12,24,25]$.

The unimodular affine normal is defined next by the expression

$$
N_{u a}=\frac{1}{2} \Delta\left(X_{0}\right),
$$

where $\Delta$ is the Laplacian operator with respect to the pseudometric $I_{u a}$; that is,

$$
\begin{aligned}
\Delta X_{0}= & \frac{1}{\sqrt{|g|}} \\
& \times \sum_{\alpha, \beta=1}^{2} \frac{\partial}{\partial u^{\alpha}}\left(\sqrt{|g|} \sum_{\beta=1}^{2} g^{\alpha \beta} \frac{\partial X_{0}}{\partial u^{\beta}}\right) \quad \text { with } g=\operatorname{det}\left(g_{\alpha \beta}\right) .
\end{aligned}
$$

It is clear that, from the above developments, we obtain three connections defined on the immersed middle surface:

(1) the Levi-Civita connection with respect to the Euclidean metric $I_{e u}$, which we shall label here as $\nabla_{\mathrm{EU}}$ and which coincides with the projection over $M_{0}$ of the usual, flat connection $D$ of $\mathbb{R}^{3}$ in the direction of the classical Euclidean normal $N_{\mathrm{EU}}$,

(2) the Levi-Civita connection with respect to the pseudometric $I_{u a}, \widetilde{\nabla}$,

(3) the affine normal induced connection, $\nabla$, that is, the projection of $D$ in the direction of the affine normal $N_{u a}$. Thus, for example, for vector fields $X$ and $Y$ tangent to $M_{0}$ we may write

$$
\nabla_{X_{p}} Y=\operatorname{proy}_{N_{u a}}\left(D_{X_{p}} Y\right) \text {. }
$$

We define next the unimodular affine second fundamental form $[7,8,24,25]$

$$
\nabla\left(I_{u a}\right):=I I_{u a}
$$

that we may also represent in local coordinates by

$$
I I_{u a}=\sum_{\alpha \beta \gamma} g_{\alpha \beta \gamma} d u^{\alpha} d u^{\beta} d u^{\gamma},
$$

with the coefficients $g_{\alpha \beta \gamma}$ totally symmetric in their indices. Some authors prefer to refer to the latter as the cubic form $[11,12]$.

Finally, we consider the affine third fundamental form, which we can describe in the following way: similar to the Euclidean case regarding Weingarten's equation, it turns out too, in affine geometry of surfaces, that the local derivatives of the affine normal belong to the tangent plane of the surface at each point; that is, we can write

$$
\frac{\partial N_{u a}}{\partial u^{\alpha}}=-\sum_{\beta} B_{\alpha}^{\beta} \frac{\partial X_{0}}{\partial u^{\beta}}=-B_{\alpha}^{1} \frac{\partial X_{0}}{\partial u^{1}}-B_{\alpha}^{2} \frac{\partial X_{0}}{\partial u^{2}}
$$

and define the affine third fundamental form by the expression:

$$
I I I_{u a}=\sum_{\alpha, \beta} B_{\alpha \beta} d u^{\alpha} d u^{\beta}, \quad \text { with } B_{\alpha \beta}=\sum_{\gamma} g_{\alpha \gamma} B_{\beta}^{\gamma} .
$$

Similarly, Weingarten's affine shape operator $S$ is defined by $[7,12]$

$$
S=S_{u a}=-\sum_{\alpha, \beta} B_{\alpha}^{\beta} d u^{\alpha} \otimes \frac{\partial}{\partial u^{\beta}} .
$$

As we have previously seen, the definition of shell as a three-dimensional body and, in particular, the Riemannian structure induced on that object by the ambient space metric is generated in a natural fashion. In the present case of unimodular affine geometry that extension is not at all that immediate. However, as we shall see, it can also be realized in a canonical way.

Proposition 1. With the above conditions and notations, one can assert that there exists a positive $h>0$ such that the given pseudometric, originally defined on the middle surface $M_{0}$, may be extended to all points at affine distance less than or equal to $h$ from $M_{0}$.

Proof. By starting from the unimodular affine invariant pseudometric $I_{u a}$, defined on the middle surface $M_{0}$,

$$
g_{\alpha \beta}=I_{u a}\left(\frac{\partial X_{0}}{\partial u^{\alpha}}, \frac{\partial X_{0}}{\partial u^{\beta}}\right),
$$

we seek, in the present context, to define on the shell a pseudometric, which is a unimodular affine invariant, to be denoted by

$$
G=\sum G_{i j} d u^{i} d u^{j}
$$

That is,

$$
G_{i j}:=G\left(\frac{\partial X}{\partial u^{i}}, \frac{\partial X}{\partial u^{j}}\right) .
$$


Observe that, since bilinearity must be preserved, we have to write in affine normal coordinates of the shell

$$
\begin{aligned}
G_{\alpha \beta} & =G\left(\frac{\partial X}{\partial u^{\alpha}}, \frac{\partial X}{\partial u^{\beta}}\right) \\
& =G\left(\frac{\partial X_{0}}{\partial u^{\alpha}}+u \frac{\partial N_{u a}}{\partial u^{\alpha}}, \frac{\partial X_{0}}{\partial u^{\beta}}+u \frac{\partial N_{u a}}{\partial u^{\beta}}\right),
\end{aligned}
$$

whence, by definition, we should have

$$
G_{\alpha \beta}:=g_{\alpha \beta}-2 u B_{\alpha \beta}+u^{2} \sum_{\lambda} B_{\alpha}^{\lambda} B_{\beta \lambda}
$$

and where, as stated previously, Greek indices run from 1 to 2. Thus, in order to extend that definition to the third index, we also write

$$
G_{3 \alpha}=G_{\alpha 3}=G\left(\frac{\partial X}{\partial u^{\alpha}}, \frac{\partial X}{\partial u^{3}}\right)=G\left(X_{\alpha}, N_{u a}\right):=0
$$

and, finally,

$$
G_{33}=G\left(\frac{\partial X}{\partial u^{3}}, \frac{\partial X}{\partial u^{3}}\right)=G\left(N_{u a}, N_{u a}\right):=1 .
$$

Then, it is easy to see that there exists $h>0$ such that, for $|u|=\left|u^{3}\right|<h$, it holds that

$$
\operatorname{det}\left(G_{i j}\right) \neq 0
$$

and, consequently, the latter is a pseudo-Riemannian, unimodular affine invariant metric defined on the shell with the suitable data, as it was our purpose to construct.

\section{Compatibility Conditions [24]}

One of the main aspects in the theory of shells is the determination of compatibility conditions. These are restraints on the behavior of the various difference tensors that can be defined by comparing the two states of the shell. The natural tool here is represented by the integrability conditions that must be satisfied, in all cases, by both middle surfaces. These conditions are very well known in the case of Euclidean shells (see, e.g., $[22,23,31,32,36]$ ) and can be described, in our notation, as follows.

For the strain tensor with components defined by $\varepsilon_{\alpha \beta}=$ $(1 / 2)\left(a_{\alpha \beta}^{*}-a_{\alpha \beta}\right)$, it is proven that

$$
\begin{aligned}
\varepsilon_{\beta, \delta}^{\beta, \delta}-\varepsilon_{\beta, \delta}^{\delta, \beta}= & L_{\beta}^{* \delta} L_{\delta}^{* \beta}-L_{\delta}^{* \delta} L_{\beta}^{* \beta} \\
& +g_{\mu}^{* \beta}\left(L_{\delta}^{\delta} L_{\beta}^{\mu}-L_{\beta}^{\delta} L_{\delta}^{\mu}\right) \\
& -g_{\mu \nu}^{*} g^{\beta \alpha} g^{\delta \gamma}\left(C_{\alpha \beta}^{\mu} C_{\gamma \delta}^{\nu}-C_{\alpha \delta}^{\mu} C_{\beta \gamma}^{\nu}\right)
\end{aligned}
$$

while for the difference tensor $w_{\alpha \beta}:=L_{\alpha \beta}^{*}-L_{\alpha \beta}$ it holds that

$$
\begin{aligned}
w_{\beta, \gamma}^{\alpha}-w_{\gamma, \beta}^{\alpha} & =g^{\alpha \rho}\left(w_{\rho \beta, \gamma}-w_{\rho \gamma, \beta}\right) \\
& =g^{\alpha \rho}\left(C_{\rho \gamma}^{\mu} L_{\mu \beta}^{*}-C_{\rho \beta}^{\mu} L_{\mu \gamma}^{*}\right) .
\end{aligned}
$$

In both equations the symbol $C_{\rho \beta}^{\mu}$ represents the components of the difference tensor between the Levi-Civita connection of $M_{0}^{*}$ and that of $M_{0}$.

Now, for the case of affine shells the corresponding compatibility conditions were obtained in our previous article [24], where the basis for our analysis and development was mainly given by the following two lemmas, with somewhat more general validity and context.

Lemma 2 (relation between the Levi-Civita connections). Let $(M, g)$ and $\left(M, g^{*}\right)$ be (pseudo-) Riemannian structures defined on the differentiable manifold $M$, denote with $\nabla$ and $\nabla^{*}$ the Levi-Civita connections associated with them, and let $\Gamma_{B C}^{A}$ and $\Gamma_{B C}^{* A}$ be the corresponding Christoffel symbols. Then, both connections are related by the equation

$$
\Gamma_{B C}^{* A}=\Gamma_{B C}^{A}+T_{B C}^{A},
$$

where $T_{B C}^{A}$ represent the components of the tensor defined by

$$
T_{B C}^{A}=\frac{1}{2} \bar{g}^{* A R}\left(g_{R B ; C}^{*}+g_{R C ; B}^{*}-g_{B C ; R}^{*}\right),
$$

and $\bar{g}^{* U V}$ are the components of the tensor which represents the inverse matrix of $g_{U V}^{*}$.

Lemma 3 (relation between the curvature tensors). In the conditions of the previous lemma, the components of the respective associated tensors of the Riemannian curvature, $R_{M N P}^{L}$ and $R_{A B C D}^{*}$, are related by the following equation:

$$
\begin{aligned}
R_{A C D B}^{*}= & \varepsilon_{A B ; C D}+\varepsilon_{C D ; A B}-\varepsilon_{A D ; ; C B}-\varepsilon_{B C ; ; A D} \\
& -\frac{1}{2}\left(g_{A M}^{*} R_{C B D}^{M}+g_{C M}^{*} R_{A D B}^{M}\right) \\
& +g_{L S}^{*}\left(T_{A B}^{L} T_{C D}^{S}-T_{A D}^{L} T_{B C}^{S}\right),
\end{aligned}
$$

where, by definition, $\varepsilon_{A B}:=(1 / 2)\left(g_{A B}^{*}-g_{A B}\right)$, and the double semicolon denotes the "second covariant derivative" with respect to the Levi-Civita connection associated with the metric $g$.

Then, with the help of those two previous lemmas, the main result of this section may be stated as follows.

Theorem 4 (compatibility conditions for affine shells). For the difference tensors defined by the various expressions that establish comparisons between the respective components of the first, second, and third fundamental forms, that is,

$$
\begin{gathered}
\varepsilon_{\alpha \beta}=\frac{1}{2}\left(g_{\alpha \beta}^{*}-g_{\alpha \beta}\right), \quad \sigma_{\alpha \beta \gamma}:=g_{\alpha \beta \gamma}^{*}-g_{\alpha \beta \gamma}, \\
w_{\alpha \beta}:=B_{\alpha \beta}^{*}-B_{\alpha \beta},
\end{gathered}
$$


and the tensor defined by comparison between the corresponding Levi-Civita connections, represented by equation

$$
\widetilde{\Gamma}_{\alpha \beta}^{* \mu}=C_{\alpha \beta}^{\mu}+\widetilde{\Gamma}_{\alpha \beta}^{\mu},
$$

there hold the following conditions:

(1) affine Gauss condition

$$
\begin{aligned}
\varepsilon_{\beta, \delta}^{\beta, \delta}-\varepsilon_{\beta, \delta}^{\delta, \beta}= & \frac{1}{2}\left(B_{\beta}^{* \beta} g_{\delta}^{* \delta}-B_{\delta}^{* \beta} g_{\beta}^{* \delta}+B_{\delta}^{* \delta} g_{\beta}^{* \beta}-B_{\beta}^{* \delta} g_{\delta}^{* \beta}\right) \\
& -\frac{1}{2} g^{\beta \alpha} g^{\delta \gamma}\left(A_{\gamma \beta}^{* \eta} \cdot g_{\alpha \eta \delta}^{*}-A_{\gamma \delta}^{* \eta} \cdot g_{\alpha \eta \beta}^{*}\right) \\
& +\frac{1}{2} g_{\mu}^{* \beta}\left(-B_{\delta}^{\delta} \delta_{\beta}^{\mu}+A_{\beta}^{\delta \eta} \cdot A_{\eta \delta}^{\mu}\right) \\
& -g_{\lambda \mu}^{*} g^{\beta \alpha} g^{\delta \gamma}\left(C_{\alpha \beta}^{\lambda} C_{\gamma \delta}^{\mu}-C_{\alpha \delta}^{\lambda} C_{\beta \gamma}^{\mu}\right)
\end{aligned}
$$

(2) affine Mainardi-Codazzi condition

$$
\begin{aligned}
\sigma_{\alpha \beta \gamma, \delta}-\sigma_{\alpha \beta \delta, \gamma}= & g_{\mu \beta \gamma}^{*} C_{\alpha \delta}^{\mu}+g_{\mu \alpha \gamma}^{*} C_{\beta \delta}^{\mu}-g_{\mu \beta \delta}^{*} C_{\alpha \gamma}^{\mu} \\
& -g_{\mu \alpha \delta}^{*} C_{\beta \gamma}^{\mu}+B_{\alpha \delta}^{*} g_{\beta \gamma}^{*}+B_{\beta \delta}^{*} g_{\alpha \gamma}^{*} \\
& -B_{\alpha \gamma}^{*} g_{\beta \delta}^{*}-B_{\beta \gamma}^{*} g_{\alpha \delta}^{*}-B_{\alpha \delta} g_{\beta \gamma} \\
& -B_{\beta \delta} g_{\alpha \gamma}+B_{\alpha \gamma} g_{\beta \delta}+B_{\beta \gamma} g_{\alpha \delta}
\end{aligned}
$$

(3) Codazzi condition for the affine shape operators

$$
\begin{aligned}
& w_{\beta, \alpha}^{\alpha}-w_{\alpha, \beta}^{\alpha} \\
& \quad=g^{\alpha \rho}\left[B_{\beta \mu}^{*}\left(C_{\rho \alpha}^{\mu}+A_{\rho \alpha}^{* \mu}\right)-B_{\alpha \mu}^{*}\left(C_{\rho \beta}^{\mu}+A_{\rho \beta}^{* \mu}\right)+B_{\alpha \mu} A_{\rho \beta}^{\mu}\right] .
\end{aligned}
$$

\section{Basic Inequalities for Affine Shells}

The following basic inequalities, involving the geometrical objects treated above, were previously obtained and may be seen in [25].

When represented in the form of Monge's, that is, as a graph, the middle surface of the shell $M_{0}$ has all of its geometrical properties related to a given function $f$ assumed to be differentiable enough and, in the present context of affine geometry, satisfying a partial differential equation of MongeAmpère type:

$$
\operatorname{det}\left(\partial_{\alpha \beta} f\right)= \pm F,
$$

and for such a kind of equations, with boundary conditions as in the present case, there hold bounds for the function $f$ and its derivatives. Also, since the function $F$ is strictly positive in the complete domain where $f$ is defined, there exist lower and upper bounds for $F$ as well.
As a consequence, we can also assume that the second derivatives of $f$ are bounded, that is, the components of the Hessian matrix $\left(\partial_{\alpha \beta} f\right)$, as well as the components of the inverse matrix of the latter, denoted by $\left(f^{\alpha \beta}\right)$, and also the components of the pseudometric tensor, covariant as well as contravariant, that is, $g_{\alpha \beta}$ and $g^{\alpha \beta}$. These facts are expressed in the following inequalities:

$$
\left|\partial_{\alpha \beta} f\right|<K, \quad\left|f^{\alpha \beta}\right|<K, \quad\left|g_{\alpha \beta}\right|<K, \quad\left|g^{\alpha \beta}\right|<K .
$$

Besides, since the higher order derivatives are also bounded and in order to unify notation, we shall assume that there exists a generalized affine upper bound of curvature, intimately related to the upper bound for the affine principal curvatures of the middle surface $M_{0}$, which we shall also denote by $R$ and which for the present, affine case remains specified by the conditions that

$$
\left|\partial_{\alpha \beta \gamma} f\right|<\frac{1}{R^{1 / 2}}
$$

and for the successive derivatives,

$$
\begin{gathered}
\left|\partial_{\alpha \beta \gamma \eta} f\right| \leq R^{-1}, \\
\left|\partial_{\alpha \beta \gamma \eta \lambda} f\right| \leq\left(R^{-1 / 2}\right)^{3}, \ldots,
\end{gathered}
$$

are satisfied for as high order of derivatives as needed in the development of the theory.

By using these hypotheses one obtains the corresponding bounds for the components of the tensor representing the third fundamental form

$$
\begin{aligned}
B_{\alpha \beta}=-\frac{1}{4}( & \partial_{\alpha \beta}(\log F)+\frac{1}{4} \partial_{\alpha}(\log F) \partial_{\beta}(\log F) \\
& \left.-\sum_{\sigma, \lambda} f^{\sigma \lambda} \partial_{\alpha \beta \sigma} f \partial_{\lambda}(\log F)\right),
\end{aligned}
$$

if we have in mind, besides, the two following, well-known identities:

$$
\begin{gathered}
\partial_{\alpha} \log F=\sum_{\rho, \sigma} f^{\rho \sigma} \partial_{\alpha \rho \sigma} f \\
\partial_{\alpha \beta} \log F=\sum_{\rho, \sigma} f^{\rho \sigma} \partial_{\alpha \beta \rho \sigma} f-\sum_{\rho, \sigma} f^{\rho \theta} f^{\tau \sigma} \partial_{\theta \tau \alpha} f \partial_{\rho \sigma \beta} f,
\end{gathered}
$$

with which it turns out that

$$
\left|B_{\alpha \beta}\right| \leq \frac{1}{4} \frac{1}{R}\left(4 K+32 K^{2}\right)=\left(K+8 K^{2}\right) \frac{1}{R} .
$$


We compute next the partial derivatives of these components:

$$
\begin{gathered}
\partial_{\gamma} B_{\alpha \beta} \\
=-\frac{1}{4}\left(\begin{array}{c}
\partial_{\gamma} f^{\rho \tau} \partial_{\alpha \beta \rho \tau} f+f^{\rho \tau} \partial_{\alpha \beta \rho \tau} f \\
-\left(\partial_{\gamma} f^{\rho \eta} \partial_{\eta \mu \alpha} f\right)\left(f^{\mu \tau} \partial_{\beta \rho \tau} f\right) \\
-\left(f^{\rho \eta} \partial_{\eta \mu \alpha \gamma} f\right)\left(f^{\mu \tau} \partial_{\beta \rho \tau} f\right) \\
-\left(f^{\rho \eta} \partial_{\eta \mu \alpha} f\right)\left(\partial_{\gamma} f^{\mu \tau} \partial_{\beta \rho \tau} f\right) \\
-\left(f^{\rho \eta} \partial_{\eta \mu \alpha} f\right)\left(f^{\mu \tau} \partial_{\beta \rho \tau} f\right)
\end{array}\right) \\
+\frac{1}{16}\left(\begin{array}{c}
\left(\partial_{\gamma} f^{\rho \tau} \partial_{\tau \rho \alpha} f\right)\left(f^{\sigma \lambda} \partial_{\beta \sigma \lambda} f\right) \\
+\left(f^{\rho \tau} \partial_{\tau \rho \alpha \gamma} f\right)\left(f^{\sigma \lambda} \partial_{\beta \sigma \lambda} f\right) \\
+\left(f^{\rho \tau} \partial_{\tau \rho \alpha} f\right)\left(\partial_{\gamma} f^{\sigma \lambda} \partial_{\beta \sigma \lambda} f\right) \\
+\left(f^{\rho \tau} \partial_{\tau \rho \alpha} f\right)\left(f^{\sigma \lambda} \partial_{\beta \sigma \lambda \lambda} f\right)
\end{array}\right) \\
-\frac{1}{4}\left(\begin{array}{c}
\left(\partial_{\gamma} f^{\sigma \lambda} \partial_{\alpha \beta \sigma} f\right)\left(f^{\rho \tau} \partial_{\lambda \rho \tau} f\right) \\
+\left(f^{\sigma \lambda} \partial_{\alpha \beta \sigma \gamma} f\right)\left(f^{\rho \tau} \partial_{\lambda \rho \tau} f\right) \\
+\left(f^{\sigma \lambda} \partial_{\alpha \beta \sigma} f\right)\left(\partial_{\gamma} f^{\rho \tau} \partial_{\lambda \rho \tau} f\right) \\
+\left(f^{\sigma \lambda} \partial_{\alpha \beta \sigma} f\right)\left(f^{\rho \tau} \partial_{\lambda \rho \tau \gamma} f\right)
\end{array}\right) .
\end{gathered}
$$

Then, by using the identity

$$
\sum_{\lambda} f^{\lambda \sigma} \partial_{\lambda \mu} f=\delta_{\mu}^{\sigma}
$$

from which it follows that

$$
\partial_{\alpha} f^{\sigma \gamma}=-\sum_{\lambda} f^{\lambda \gamma} f^{\mu \sigma} \partial_{\alpha \lambda \mu} f
$$

we find by direct computation the following estimate:

$$
\left|\partial_{\gamma} B_{\alpha \beta}\right| \leq \frac{1}{R^{3 / 2}}\left(K+19 K^{2}+24 K^{3}\right) .
$$

With the development done so far, we can also obtain estimates for the components of the pseudometric, that is, the components of the pseudo-Riemannian tensor $G=$ $\sum_{i, j} G_{i j} d u^{i} d u^{j}$ of the shell in the undeformed state and its successive derivatives, partial as well as covariant. For example, we observe first from the very definition that

$$
G_{\alpha \beta}=g_{\alpha \beta}-2 u B_{\alpha \beta}+u^{2} \sum_{\lambda, \mu} g^{\lambda \mu} B_{\alpha \mu} B_{\beta \lambda}
$$

and, consequently, obtain

$$
\left|G_{\alpha \beta}\right| \leq K+2 K(1+8 K) \frac{h}{R}+4 K^{2}(1+8 K)\left(\frac{h}{R}\right)^{2} .
$$

In a similar fashion, one can obtain other estimates for the higher order derivatives. All of these estimates shall be used in the attainment of one of our important objectives: to find estimates for the higher order covariant derivatives for the strain and the stress tensors.

\section{Strain-Stress Relations in Euclidean and Affine Shells}

For the case of Affine shells, the contravariant components of the stress tensor, $t^{m k}$, are connected with the components of the strain tensor, $\varepsilon_{m k}$, by means of the stress-strain relations

$$
t^{m k}:=\sqrt{\frac{G}{G^{*}}} \frac{\partial W}{\partial \varepsilon_{m k}}
$$

which are defined in a similar fashion as to the Euclidean case, introduced by John $[22,23]$. In the latter equation $W$ is the strain energy density of the given material that relates both the stress and the strain tensors, defined by

$$
\begin{gathered}
t:=\sum_{i, j} t_{i j} d u^{i} d u^{j}=\sum_{i, j} t^{i j} \frac{\partial}{\partial u^{i}} \otimes \frac{\partial}{\partial u^{j}}, \\
\varepsilon:=\sum_{i, j} \varepsilon_{i j} d u^{i} d u^{j},
\end{gathered}
$$

with $\varepsilon_{i j}=(1 / 2)\left(G_{i j}^{*}-G_{i j}\right)$.

The same expression in terms of the corresponding $(1,1)$ tensors is

$$
t_{i}^{m}=\sum_{k} G_{i k} t^{m k}=\sqrt{\frac{G}{G^{*}}} \frac{\partial W}{\partial \varepsilon_{m}^{i}} .
$$

Next, we introduce the components of the "pseudo-stress tensor" defined by

$$
T_{i}^{m}:=\sqrt{\frac{G^{*}}{G}} t_{i}^{m}-\delta_{i}^{m} W,
$$

and we may also write

$$
\begin{aligned}
T_{i}^{m}= & \left(W_{s_{1}}-W\right) \delta_{i}^{m}+\left(2 W_{s_{1}}+2 W_{s_{2}}\right) \varepsilon_{i}^{m} \\
& +\left(4 W_{s_{2}}+3 W_{s_{3}}\right) \sum_{k} \varepsilon_{i}^{k} \varepsilon_{k}^{m}+6 W_{s_{3}} \sum_{s, k} \varepsilon_{i}^{s} \varepsilon_{s}^{k} \varepsilon_{k}^{m},
\end{aligned}
$$

where

$$
\begin{gathered}
s_{1}=\sum_{i} \varepsilon_{i}^{i}, \\
s_{2}=\sum_{i, j} \varepsilon_{j}^{i} \varepsilon_{i}^{j}, \\
s_{3}=\sum_{i, j, k} \varepsilon_{j}^{i} \varepsilon_{k}^{j} \varepsilon_{i}^{k} .
\end{gathered}
$$

Then, the equations of equilibrium [25] can be written:

$$
t_{, j}^{i j}+c_{h j}^{i} t^{h j}+c_{h j}^{h} t^{i j}=0,
$$

where the double comma ,, indicates covariant differentiation with respect to the Levi-Civita connection determined by the pseudo-Riemannian structure $G$ and where

$$
c_{j k}^{i}=\frac{1}{2} \bar{G}^{* i r}\left(G_{r j, k}^{*}+G_{r k, j}^{*}-G_{j k, r}^{*}\right) .
$$


Therefore, we also have as a consequence that

$$
\begin{aligned}
\sum_{m} T_{i ; m}^{m}= & \left(\sqrt{\frac{G^{*}}{G}}\right) \\
& \times \sum_{m, r, s}\left(c_{m r}^{r} t^{m s} G_{s i}^{*}-t^{r s} c_{r m}^{m} G_{s i}^{*}\right. \\
& \left.-t^{m r} c_{r m}^{s} G_{s i}^{*}+t^{m s} G_{s i ; m}^{*}-\frac{1}{2} t^{m s} G_{s m ; i}^{*}\right)=0 .
\end{aligned}
$$

Additional notations are needed in order to compare the components of the stress and strain tensors, even those belonging to different spaces of definition, for example, and very particularly, in order to compare the components of type $(0,2)$ tensors $t^{i j}$ with those with components of type $(1,1) t_{i}^{j}$. Thus, we follow in this respect the kind of notation previously introduced by John in $[22,23]$, in particular the so-called "general form of an expression" like

$$
F(p, q)(u+v+w)
$$

representing a vector in a suitable space, where $u, v, w, p$, and $q$ are vectors themselves. The notation indicates that each of the components of $F(p, q)(u+v+\omega)$ is a sum of a linear form in the components of $u$, a linear form in the components of $v$, and a linear form in the components of $w$. The coefficients of these linear forms are functions of the components of the vectors $p$ and $q$ defined and differentiable as often as needed for all sufficiently small "lengths" $|p|$ and $|q|$. The letter $F$ stands for a different expression in every equation to be considered. Thus, for example, we can write for the components of the stress and strain tensors of type $(1,1)$

$$
t=\left(t_{k}^{i}\right), \quad \varepsilon=\left(\varepsilon_{k}^{i}\right) .
$$

Thus, in terms of the Lamé coefficients $\lambda$, and $\mu$, the following equation is valid:

$$
t_{i}^{m}=\lambda \sum_{j} \varepsilon_{j}^{j} \delta_{i}^{m}+2 \mu \varepsilon_{i}^{m}+F(\varepsilon) \varepsilon^{2}
$$

since such coefficients are defined by the relation

$$
W:=\frac{\lambda}{2}\left(s_{1}\right)^{2}+\mu s_{2}+F(\varepsilon) \varepsilon^{3}
$$

where, as before,

$$
s_{1}=\sum_{i} \varepsilon_{i}^{i}, \quad s_{2}=\sum_{i, j} \varepsilon_{j}^{i} \varepsilon_{i}^{j}, \quad s_{3}=\sum_{i, j, k} \varepsilon_{j}^{i} \varepsilon_{k}^{j} \varepsilon_{i}^{k}
$$

and where we observe that the first two terms, on the righthand side, are quadratic with regards to the strain tensor (operator) $\varepsilon=\left(\varepsilon_{k}^{i}\right)$, while the third term involves all of those components of order higher than two, representing otherwise the "remainder," of paramount importance when coming to the corresponding numerical estimates.
From now on we establish that in the same sense all of the expressions to follow have to be interpreted.

Hence, by taking partial derivatives, we can write

$$
\begin{gathered}
W_{s_{1}}=\frac{\partial W}{\partial s_{1}}=\partial_{s_{1}} W=\lambda s_{1}, \\
W_{s_{2}}=\frac{\partial W}{\partial s_{2}}=\partial_{s_{2}} W=\mu .
\end{gathered}
$$

From the latter we obtain, successively, first that

$$
\begin{gathered}
2 W_{s_{1}}+2 W_{s_{2}}=2 \mu+2 \lambda s_{1}, \\
4 W_{s_{2}}+3 W_{s_{3}}=2 \mu+F(t) t^{3}, \\
W_{s_{1}}-W=\lambda s_{1}-\frac{1}{2} \lambda\left(s_{1}\right)^{2}-\frac{1}{2} \mu s_{2}+F(\varepsilon) \varepsilon^{3} .
\end{gathered}
$$

Then, by using Taylor's series development

$$
(1+x)^{-1 / 2}=1+\left(-\frac{1}{2}\right) x+\frac{3 / 4}{2} x^{2}+\cdots,
$$

we can express

$$
\sqrt{\frac{G}{G^{*}}}=\left(\frac{G}{G^{*}}\right)^{1 / 2}=\left(\frac{G^{*}}{G}\right)^{-1 / 2}=1-\frac{1}{2} s_{1}+\frac{3}{8}\left(s_{1}\right)^{2}+\cdots,
$$

and also

$$
t_{i}^{m}=\sqrt{\frac{G}{G^{*}}}\left(W_{s_{1}} \delta_{i}^{m}+2 W_{s_{2}} \varepsilon_{i}^{m}+3 W_{s_{3}} \sum_{j} \varepsilon_{j}^{m} \varepsilon_{i}^{j}\right)
$$

becomes, first,

$$
\begin{aligned}
t_{i}^{m}= & \left(1-\frac{1}{2} s_{1}+\frac{3}{8}\left(s_{1}\right)^{2}+\cdots\right) \\
& \times\left(W_{s_{1}} \delta_{i}^{m}+2 W_{s_{2}} \varepsilon_{i}^{m}+3 W_{s_{3}} \sum_{j} \varepsilon_{j}^{m} \varepsilon_{i}^{j}\right)
\end{aligned}
$$

and, afterwards,

$$
\begin{aligned}
t_{i}^{m} & =W_{s_{1}} \delta_{i}^{m}+2 W_{s_{2}} \varepsilon_{i}^{m}+3 W_{s_{3}} \sum_{j} \varepsilon_{j}^{m} \varepsilon_{i}^{j} \\
& =\frac{\lambda}{2} s_{1} \delta_{i}^{m}+2 \mu \varepsilon_{i}^{m}+3 W_{s_{3}} \sum_{j} \varepsilon_{j}^{m} \varepsilon_{i}^{j} \\
& =\frac{\lambda}{2} \sum_{j} \varepsilon_{j}^{j} \delta_{i}^{m}+2 \mu \varepsilon_{i}^{m}+F(\varepsilon) \varepsilon^{2} .
\end{aligned}
$$

From the latter, the trace of the stress tensor (operator) can be written:

$$
\sum_{j} t_{j}^{j}=\left(\frac{3}{2} \lambda+2 \mu\right) \sum_{j} \varepsilon_{j}^{j}+F(\varepsilon) \varepsilon^{2},
$$

where $\sum_{j} \varepsilon_{j}^{j}$ itself represents the trace of the strain tensor (operator). 
Hence, from the above we can also write

$$
\varepsilon_{i}^{m}=\frac{1}{2 \mu} t_{i}^{m}-\frac{\lambda}{2 \mu} s_{1} \delta_{i}^{m}+F(t) t^{2}
$$

or, also,

$$
\varepsilon_{i}^{m}=\frac{1}{2 \mu} t_{i}^{m}-\frac{1-2 \mu}{2 \mu} \sum_{j} t_{j}^{j} \delta_{i}^{m}+F(t) t^{2} .
$$

Then, the expression for the components of the pseudostress tensor is

$$
\begin{aligned}
T_{i}^{m}= & t_{i}^{m}+\frac{2}{\mu} \sum_{i} t_{i}^{m} t_{i}^{s}+\left(\frac{5 \mu-2}{\mu}\right) \sum_{j} t_{j}^{j} t_{i}^{s} \\
& -\frac{1}{2}\left(\frac{1}{2 \mu} \sum_{r, s} t_{s}^{r} t_{r}^{s}-\left(\frac{1-2 \mu}{2 \mu}\right) \sum_{r} t_{r}^{r} \sum_{s} t_{s}^{s}\right) \delta_{i}^{m}+F(t) t^{3} .
\end{aligned}
$$

We introduce next the "vector" $\eta=\left(\eta_{k}^{i}\right)$ by means of the following relation:

$$
G_{i k}=\delta_{k}^{i}+\eta_{k}^{i},
$$

which clearly measures the difference between the metric matrix and that corresponding to the identity.

Then, we obtain the following estimate for the components of the corresponding inverse matrix $\left(G^{i k}\right):=\left(G_{i k}\right)^{-1}$ :

$$
G^{i k}=\delta_{k}^{i}+\eta_{k}^{i}+F(\eta)\left(\eta^{2}\right) .
$$

Next, a straightforward computation shows that the Christoffel symbols associated with the Levi-Civita connection determined by the pseudometric $G$ satisfy the estimate

$$
\Gamma_{k r}^{i}=F(\eta)\left(\eta^{\prime}\right) .
$$

Then, it also holds the following estimate:

$$
t_{i k}=t_{k}^{i}+F(\eta, t)(\eta t) .
$$

For the metric tensor in the deformed "strained" state we have, by definition,

$$
G_{i k}^{*}=G_{i k}+2 \varepsilon_{i k} .
$$

Hence, we can also estimate that

$$
\begin{aligned}
G_{i k}^{*}= & G_{i k}+\frac{2}{\mu} t_{i k} \\
& -2\left(\frac{1-2 \mu}{2 \mu}\right)\left(\sum_{j} t_{j}^{j}\right) \delta_{i k}+F(t, \eta)\left(t^{2}+\eta t\right) .
\end{aligned}
$$

For the tensor with components $c_{k r}^{i}$ measuring the change in the Levi-Civita connections, from the "unstrained" natural state to the deformed "strained" state, we estimate

$$
c_{k r}^{i}=F(\eta, t)\left(t^{\prime}+\eta^{\prime} t\right) .
$$

Then, we also obtain the two following estimates:

$$
\begin{gathered}
\sum_{m} t_{i m ; m}=F(\eta, t)\left(t t^{\prime}+\eta^{\prime} t+\eta t^{\prime}\right), \\
\sum_{r} t_{h k ; r r}+2 \mu \sum_{r} t_{r r ; h k} \\
=F(\eta, t)\left(\eta t^{\prime \prime}+\left(t^{\prime}\right)^{2}+t \eta^{\prime} t^{\prime}+\eta^{\prime} t^{\prime}+\eta^{\prime \prime} t\right. \\
\left.+\left(\eta^{\prime}\right)^{2} t+\left(\eta^{\prime}\right)^{2} t^{2}+t t^{\prime \prime}\right),
\end{gathered}
$$

since, in the present case, the three-dimensional compatibility equations are given in terms of the comparison between the Riemannian curvature tensors of the affine shell, when passing from the natural (undeformed) to the deformed state.

Let us remark here on an important comparison that holds between the expression obtained by John [22], in Euclidean geometry, by using the corresponding compatibility conditions, which in that context furnish for both states of the shell that the curvature tensor is vanishing, as expressed in (7) of the cited article; that is,

$$
\begin{aligned}
0=R_{a c d b}^{*}= & \varepsilon_{a b ; c d}+\varepsilon_{c d ; a b}-\varepsilon_{a d ; c b} \\
& -\varepsilon_{b c ; a d}+\sum_{l, s} G_{l s}^{*}\left(c_{a b}^{l} c_{c d}^{s}-c_{a d}^{l} c_{b c}^{s}\right),
\end{aligned}
$$

while for us, in the present context of affine geometry, the same kind of geometrical object is in general not vanishing, so that we have to include both by writing

$$
\begin{aligned}
R_{a c d b}^{*}= & \varepsilon_{a b ; c d}+\varepsilon_{c d ; a b}-\varepsilon_{a d ; c b}-\varepsilon_{b c ; a d} \\
& -\frac{1}{2}\left(\sum_{m} G_{a m}^{*} R_{c b d}^{m}+\sum_{m} G_{c m}^{*} R_{a d b}^{m}\right) \\
& +\sum_{l, s} G_{l s}^{*}\left(c_{a b}^{l} c_{c d}^{s}-c_{a d}^{l} c_{b c}^{s}\right)
\end{aligned}
$$

obtained by direct application of Lemma 3 above. Because of this significant fact, both of them have to be estimated.

Thus, if we denote by $\varepsilon_{a b ; c d}$ the second covariant derivatives with respect to the Levi-Civita connection associated with the pseudometric $G$, we further obtain from the latter equation

$$
\begin{aligned}
\varepsilon_{a b ; c d}+ & \varepsilon_{c d ; a b}-\varepsilon_{a d ; c b}-\varepsilon_{b c ; a d} \\
= & -\sum_{l, s} G_{l s}^{*}\left(c_{a b}^{l} c_{c d}^{s}-c_{a d}^{l} c_{b c}^{s}\right)+R_{a c d b}^{*} \\
& -\frac{1}{2}\left(\sum_{m} G_{a m}^{*} R_{c b d}^{m}+\sum_{m} G_{c m}^{*} R_{a d b}^{m}\right),
\end{aligned}
$$

and it is not difficult to get the following estimates for those tensors:

$$
\begin{gathered}
R=F(\eta)\left(\eta^{\prime \prime}+(\eta)^{2}+\left(\eta^{\prime}\right)^{2}\right), \\
R^{*}=F(\eta, t)\left(\left(t^{\prime}\right)^{2}+\left(\eta^{\prime}\right)^{2}+t^{\prime \prime}+\eta^{\prime \prime} t+\eta^{\prime} t^{\prime}+\eta t\right) .
\end{gathered}
$$


In what follows we shall also denote by $\varepsilon$ an upper bound for the absolute values of the principal strains at all points of the shell. Let $P_{0}$ be a point on the undeformed middle surface $M_{0}$ and $D$ the closest affine distance from $P_{0}$ to the lateral surface of the shell. Also, $2 h$ represents the thickness of the shell and $R$ is the typical length associated with the middle surface, all these quantities having been previously introduced above and in our article [25].

Then, we introduce the quantity

$$
\theta=\max \left(\frac{h}{D}, \sqrt{\frac{h}{R}}, \sqrt{\varepsilon}\right)
$$

and assume, besides, that the circumstances are such that

$$
\theta<\theta_{0}
$$

where $\theta_{0}$ is a constant which depends only on the choice of the strain energy density $W$.

We assume that all of the calculations shall be done for a system of affine normal coordinates, as indicated previously and also fully described in [24].

The middle surface of the shell is represented then in the form of Monge's, that is, as a graph function, where the origin of coordinates is located precisely at $P_{0} \in M_{0}$, with the first two axes of coordinates chosen to lie at the tangent plane to $M_{0}$ at that point and with the third axis in the affine normal direction at the same point. The estimates to be computed for the partial derivatives of the function representing $M_{0}$, in the system chosen, shall be used immediately to make the corresponding estimates for the successive covariant derivatives $t_{i k ; r s \ldots}$, so that the latter estimates shall be independent of the system originally used.

Thus, we define

$$
\lambda=\frac{\theta_{0} h}{\theta}=\theta_{0} \min \left(D, \sqrt{R h}, \frac{h}{\sqrt{\varepsilon}}\right)
$$

and obtain the inequalities

$$
h<\lambda<\sqrt{R h}, \quad h<\frac{R}{4}, \quad \lambda<\frac{D}{2},
$$

which are easily seen to be satisfied if we assume, for example, that

$$
\theta_{0}<\frac{1}{2}
$$

From those we obtain that

$$
\frac{1}{R} \leq\left(\theta_{0}\right)^{2} \frac{h}{\lambda^{2}} \leq \theta_{0} \theta \frac{1}{\lambda} \leq \frac{1}{2} \frac{\theta}{\lambda}, \quad \varepsilon<\left(\theta_{0}\right)^{2} \frac{h^{2}}{\lambda^{2}}<\frac{h^{2}}{4 \lambda^{2}} .
$$

It is to be further assumed next that $\theta_{0}$ is chosen so small that for the given strain energy function $W$ all of the above formulae are valid in the region defined by

$$
M=\left\{\left(u^{1}, u^{2}, u^{3}\right): \sum\left(u^{\alpha}\right)^{2}<\lambda^{2},\left|u^{3}\right|<h\right\} .
$$

Also, from now on we shall use the same symbols of approximation as described by John [22], represented by " $O$ " and " $o$ "; that is, the first symbol is used in the conventional, classical way except that dependence on $W$ is allowed. Thus, the relation

$$
A=O(B)
$$

where $B \geq 0$, means that for a given strain energy function $W$ there exists a positive number $K$ such that

$$
|A| \leq K B
$$

The second one shall be used in an unconventional sense and only in combination with the first. The relation

$$
A=O(B)+o(C)
$$

where $B \geq 0$ and $C \geq 0$, shall mean that for a given strain energy function $W$ there exists a function $K(k)$, defined for all positive $k$ such that

$$
|A| \leq K(k) B+k C,
$$

for all $k>0$.

Besides, we shall assume that the strain energy function $W\left(s_{1}, s_{2}, s_{3}\right)$ is defined for all values of $\left|s_{i}\right|$ small enough and is as differentiable as needed. Here $s_{i}$ are the traces of the successive powers of the strain operator. By definition, the "length" of such "strain operator," (1,1)-tensor with components $\varepsilon_{i}^{m}$, is $|\varepsilon|:=\sqrt{\sum_{i, m} \varepsilon_{i}^{m} \varepsilon_{i}^{m}}$. For the pseudometric tensor $G$ sufficiently close to the unit matrix, that is, for $|\eta|$ sufficiently small, we can estimate $|\varepsilon|$ in terms of the eigenvalues of the matrix $\left(\varepsilon_{i}^{m}\right)$, that is, in terms of the so-called principal strains.

Then, there exists a positive $\varepsilon_{0}$ only depending on the choice of the strain energy function $W$ such that the strainstress relations hold for $|\varepsilon|<\varepsilon_{0}$, and it also follows that, for such values, $t_{i}^{m}=O(|\varepsilon|)$.

Hence, for a given function $W$ we can also find bounds $t_{0}$ and $\eta_{0}$ such that for $|t|<t_{0}$ and $|\eta|<\eta_{0}$ all of the previously stated estimates are valid and, besides, $|\varepsilon|<\varepsilon_{0}$.

\section{Estimates for the $L_{2}$-Norms of Second Order Derivatives}

In what follows, we shall use the following expression of the norm $\|w\|$ for any vector $w=w\left(u^{1}, u^{2}, u^{3}\right)$ defined in the working region $M$ specified above:

$$
\|w\|=\sqrt{\iiint_{M}|w| d u^{1} d u^{2} d u^{3}} .
$$

The symbol $w^{\prime}$ shall denote the gradient of $w$, that is, the vector whose components are the first derivatives of the components of $w$ with respect to $u^{1}, u^{2}$, and $u^{3}$. We shall denote, besides, with $w^{\bullet}$ the "surface" coordinates gradient of $w$, that is, the vector of first derivatives with respect to $u^{1}$ and $u^{2}$ only. 
It is well known that the components of the stress tensor $t_{i k}$ satisfy the symmetry condition

$$
t_{i k}=t_{k i} \text {. }
$$

We can represent the estimates obtained from the equations of equilibrium for the Euclidean case (see [22, 23] for full details) by

$$
\sum_{m} t_{i m ; m}=P_{i}=F(\eta, t)\left(t t^{\prime}+\eta^{\prime} t+\eta t^{\prime}\right)
$$

and the estimates resulting from the compatibility conditions [24] by

$$
\begin{aligned}
& \sum_{r} t_{h k ; r r}+2 \mu \sum_{r} t_{r r ; h k} \\
&=Q_{h k}=F(\eta, t)\left(\eta t^{\prime \prime}+\left(t^{\prime}\right)^{2}+t \eta^{\prime} t^{\prime}+\eta^{\prime} t^{\prime}\right. \\
&\left.+\eta^{\prime \prime} t+\left(\eta^{\prime}\right)^{2} t+\left(\eta^{\prime}\right)^{2} t^{2}+t t^{\prime \prime}\right)
\end{aligned}
$$

We obtain correspondingly for the affine theory of shells:

$$
\begin{aligned}
& \sum_{r} t_{h k ; r r}+2 \mu \sum_{r} t_{r r ; h k} \\
& \quad=F(\eta, t)\left(\begin{array}{c}
\left(t^{\prime}\right)^{2}+\eta^{\prime} t t^{\prime}+\left(\eta^{\prime} t\right)^{2}+\left(\eta^{\prime}\right)^{2}+t^{\prime \prime}+\eta^{\prime \prime} t \\
+\eta^{\prime} t^{\prime}+\eta t+\eta^{\prime \prime}+(\eta)^{2}+\eta^{\prime \prime} t^{2}+(\eta t)^{2} \\
+\left(\eta^{\prime} t\right)^{2}+\eta \eta^{\prime \prime} t+(\eta)^{3} t+\eta t\left(\eta^{\prime}\right)^{2}
\end{array}\right) .
\end{aligned}
$$

In fact, by using the previous estimates one may write, in a general form,

$$
\varepsilon_{a b ; c d}+\varepsilon_{c d ; a b}-\varepsilon_{a d ; c b}-\varepsilon_{b c ; a d}=\cdots \text { estimated terms, }
$$

and, consequently,

$$
\begin{aligned}
t_{h k ; r r}+ & \frac{1}{1+v} t_{r r ; h k} \\
= & \frac{v}{1+v}\left(t_{1, r r} \delta_{h}^{k}-t_{1, r k} \delta_{r}^{h}-t_{1, k r} \delta_{k}^{r}\right) \\
& +t_{h r ; k k}+t_{r k ; h r}+\text { higher order terms, }
\end{aligned}
$$

since

$$
\begin{aligned}
t_{i j ; k l}= & t_{i j, k l}-\left(\Gamma_{i k}^{h}\right)_{, l} t_{h j}-\Gamma_{i k}^{h} t_{h j, l}-\left(\Gamma_{j k}^{m}\right)_{, l} t_{i m} \\
& -\Gamma_{j k}^{m} t_{i m, l}-\Gamma_{i l}^{h}\left(t_{h k, j}-\Gamma_{h j}^{r} t_{r k}-\Gamma_{k j}^{s} t_{s h}\right) \\
& -\Gamma_{j l}^{m}\left(t_{m i, k}-\Gamma_{m k}^{r} t_{r i}-\Gamma_{i k}^{s} t_{s m}\right) \\
& -\Gamma_{k l}^{q}\left(t_{q i, j}-\Gamma_{q i}^{r} t_{r j}-\Gamma_{i j}^{s} t_{s q}\right) .
\end{aligned}
$$

Thus, by using all of the above expressions one may write, for the case of affine shells, estimates which resemble the ones obtained for the Euclidean case.
In fact, we develop estimates for the norms of the second derivatives of the (symmetric) tensor components $t_{i k}$, in terms of the above quantities $P_{i}$ and $Q_{h k}$, introducing the auxiliary function $\phi: M \rightarrow \mathbb{R}$, defined by

$$
\phi=\left(1-\lambda^{-2} u^{\alpha} u^{\alpha}\right)^{2} .
$$

One verifies easily the following properties:

$$
\begin{gathered}
0 \leq \phi \leq 1, \quad \partial_{3} \phi=0, \\
\left|\phi^{\bullet}\right|=\sqrt{\partial_{\alpha} \phi \partial_{\alpha} \phi} \leq \frac{4}{\lambda} \phi^{1 / 2}, \\
\left|\phi^{\bullet}\right|=\sqrt{\partial_{\alpha \beta} \phi \partial_{\alpha \beta} \phi} \leq \frac{4 \sqrt{10}}{\lambda^{2}} .
\end{gathered}
$$

Next, we consider the integral

$$
A=: \sqrt{\iiint_{M} \phi^{2} \partial_{i \alpha} t_{3 k} \partial_{i \alpha} t_{3 k} d u^{1} d u^{2} d u^{3}}
$$

whereas, in a similar fashion to the Euclidean case, one obtains the estimates

$$
\left\|\phi t^{\bullet \bullet}\right\|=O\left(A+\left\|\phi P^{\prime}\right\|+\|\phi Q\|+\lambda^{-2}\|t\|\right),
$$

with

$$
\begin{gathered}
t^{\bullet}=\left(\partial_{\alpha \beta} t_{i k}\right), \quad P^{\prime}=\left(\partial_{k} P_{i}\right), \quad Q=\left(Q_{i k}\right), \\
\left\|\phi t^{\prime \prime}\right\|=O\left(\lambda h^{-1}\left(A+\left\|\phi P^{\prime}\right\|+\|\phi Q\|+\lambda^{-2}\|t\|\right)\right) .
\end{gathered}
$$

Similarly, one may estimate the above expression $A$ :

$$
\begin{aligned}
A= & O\left(\lambda^{-2}\|t\|+\|\phi Q\|+\lambda h^{-1}\left\|\phi P^{\bullet}\right\|\right) \\
& +o\left(\left\|\phi t^{\bullet \bullet}\right\|+\lambda^{-1} h\left\|\phi t^{\prime \prime}\right\|\right) .
\end{aligned}
$$

Then, it follows that

$$
\begin{aligned}
& \left\|\phi t^{\bullet \bullet}\right\|=O\left(\lambda^{-2}\|t\|+\|\phi Q\|+\left\|\phi^{\bullet} t^{\bullet}\right\|^{2}+\lambda h^{-1}\left\|\phi P^{\bullet}\right\|\right), \\
& \left\|\phi t^{\prime \prime}\right\| \\
& \quad=O\left(\lambda h^{-1}\left(\lambda^{-2}\|t\|+\|\phi Q\|+\left\|\phi^{\bullet} t^{\bullet}\right\|^{2}+\lambda h^{-1}\left\|\phi P^{\bullet}\right\|\right)\right) .
\end{aligned}
$$

Next, we make use of the particular form of the second and third members in the equations above, by writing

$$
\begin{aligned}
& P_{i}=F(\eta, t)\left(t t^{\prime}+\eta^{\prime} t+\eta t^{\prime}\right), \\
& Q_{h k}=F(\eta, t)\left(\begin{array}{c}
\left(t^{\prime}\right)^{2}+\eta^{\prime} t t^{\prime}+\left(\eta^{\prime} t\right)^{2}+\left(\eta^{\prime}\right)^{2}+t^{\prime \prime}+\eta^{\prime \prime} t \\
+\eta^{\prime} t^{\prime}+\eta t+\eta^{\prime \prime}+(\eta)^{2}+\eta^{\prime \prime} t^{2}+(\eta t)^{2} \\
+\left(\eta^{\prime} t\right)^{2}+\eta \eta^{\prime \prime} t+(\eta)^{3} t+\eta t\left(\eta^{\prime}\right)^{2}
\end{array}\right),
\end{aligned}
$$

as well as on the respective (gradient) derivatives $P^{\prime}$ and $P^{\bullet}$. 
Thus, by taking into account these differences with the Euclidean case we find, by taking at the same time a sufficiently small value for the constant $\theta_{0}$, that

$$
\begin{gathered}
\left\|\phi t^{\bullet \bullet}\right\|=O\left(\lambda^{-2}\|t\|^{2}\right), \\
\left\|\phi t^{\prime \prime}\right\|=O\left(\lambda^{-1} h^{-1}\|t\|^{2}\right) .
\end{gathered}
$$

\section{Estimates for the $L_{2}$-Norms of Higher Order Derivatives}

From the previous development we observe that a similar approach can be made by considering again the differential equations involving the quantities $P_{i}$ and $Q_{h k}$ above, and by taking partial derivatives, that is, for a fixed value of $\gamma$, the $\partial_{\gamma} t_{i k}$ quantities satisfy the same equations if we substitute $P_{i}$ and $Q_{h k}$, respectively, by $\partial_{\gamma} P_{i}$ and $\partial_{\gamma} Q_{h k}$. Thus, we obtain estimates for $\left\|\phi \partial_{\gamma \alpha \beta} t_{i k}\right\|$ and $\left\|\phi \partial_{\gamma r s} t_{i k}\right\|$. On the other hand, the remaining component $\left\|\phi \partial_{333} t_{i k}\right\|$ may also be estimated if one observes that all those of the form $\partial_{333} t_{i k}$ are expressible in terms of the quantities $\partial_{\gamma r s} t_{m n}$ and suitable derivatives of the expressions $P_{i}$ and $Q_{h k}$. In such a fashion we are led to obtain estimates like the following ones:

$$
\begin{gathered}
\| \phi t^{\bullet \bullet \bullet}=O\left(\lambda^{-2}\left\|t^{\bullet}\right\|+\left\|\phi Q^{\prime}\right\|\right. \\
\left.+\left\|\phi P^{\prime \prime}\right\|+\lambda h^{-1}\left\|\phi P^{\bullet \bullet}\right\|\right), \\
\left\|\phi t^{\prime \prime \prime}\right\|=O\left(\lambda h ^ { - 1 } \left(\lambda^{-2}\left\|t^{\bullet}\right\|+\left\|\phi Q^{\prime}\right\|+\left\|\phi P^{\prime \prime}\right\|\right.\right. \\
\left.\left.+\lambda h^{-1} \| \phi P^{\bullet \bullet}\right)\right) .
\end{gathered}
$$

So far, in all of the previous development we kept the value of $\lambda$ fixed regarding the defining region $M$, the function $\phi$, and the norm $\|\omega\|$, all of those depending on $\lambda$. However, for estimating higher order terms we shall need to reduce further and further the region of work. Thus, we are led to rename all of those objects, for example, by calling $M_{\lambda}$ instead of $M, \phi_{\lambda}$ in place of $\phi$, and $\|\omega\|_{\lambda}$ for $\|\omega\|$. Moreover, we shall also proceed in our work by replacing $\lambda$ with $\lambda / 2$. Consequently, we replace too $\theta_{0}$ by $\theta_{0} / 2$; that is, in doing so we are restricted now to values $\theta$ such that $\theta<\theta_{0} / 2$. Next, we observe that $1=O\left(\phi_{\lambda}\right)$ in the region $M_{\lambda / 2}$, so that

$$
\|\omega\|_{\lambda / 2}=O\left(\left\|\phi_{\lambda} \omega\right\|_{\lambda}\right)
$$

It follows that

$$
\begin{aligned}
\| \phi_{\lambda / 2} t^{\cdots \bullet} & \|_{\lambda / 2} \\
=O & \left(\lambda^{-2}\left\|\phi_{\lambda} t^{\bullet}\right\|_{\lambda}+\left\|\phi_{\lambda / 2} Q^{\prime}\right\|_{\lambda / 2}\right. \\
& \left.+\left\|\phi_{\lambda / 2} P^{\prime \prime}\right\|_{\lambda / 2}+\lambda h^{-1}\left\|\phi_{\lambda / 2} P^{\bullet \bullet}\right\|_{\lambda / 2}\right),
\end{aligned}
$$

with the corresponding expression for $\left\|\phi_{\lambda / 2} t^{\prime \prime \prime}\right\|_{\lambda / 2}$ and also the corresponding changes for the (gradient) derivatives $P^{\prime \prime}$ and $P^{\bullet \bullet}$.
Then, a similar argument to the one in the Euclidean case allows us to conclude first that

$$
\begin{gathered}
\left\|\phi_{\lambda / 2} t^{\prime \prime \prime}\right\|_{\lambda / 2}=O\left(\lambda^{-2} h^{-1}\|t\|_{\lambda}^{2}\right), \\
\left\|\phi_{\lambda / 2} t^{\cdots}\right\|_{\lambda / 2}=O\left(\lambda^{-3}\|t\|_{\lambda}^{2}\right) .
\end{gathered}
$$

This kind of process can be repeated over and over again, with the corresponding changes. For example, by applying the above relations to $t^{\circ}$, in place of $t$, and, at the same time, replacing $\lambda$ by $\lambda / 4, P$ by $P^{\bullet}$, and $Q$ by $Q^{\bullet}$, we find that

$$
\begin{aligned}
&\left\|\phi_{\lambda / 4} t^{\cdots \cdots \bullet}\right\|_{\lambda / 4} \\
&=O\left(\lambda^{-2}\left\|\phi_{\lambda / 2} t^{\bullet \bullet}\right\|_{\lambda / 2}+\left\|\phi_{\lambda / 4} Q^{\prime \prime}\right\|_{\lambda / 4}\right. \\
&\left.\quad+\left\|\phi_{\lambda / 4} P^{\prime \prime \prime}\right\|_{\lambda / 4}+\lambda h^{-1}\left\|\phi_{\lambda / 4} P^{\cdots \cdot}\right\|_{\lambda / 4}\right),
\end{aligned}
$$

with a similar expression for the case of $\left\|\phi_{\lambda / 4} t^{\prime \prime \prime \prime}\right\|_{\lambda / 4}$.

Therefore, the above development allows stating the following preliminary result, in the form of estimates for the corresponding partial derivatives of higher order.

Lemma 5. With the above notations and conditions, there hold the estimates

$$
\begin{gathered}
\partial_{k_{1}, k_{2}, i_{1}, i_{2}, \ldots, i_{n}} t=O\left(\varepsilon^{2} \lambda^{1-n} h^{-1}\right), \\
\partial_{k_{1}, k_{2}, \alpha_{1}, \alpha_{2}, \ldots, \alpha_{n}} t=O\left(\varepsilon^{2} \lambda^{-n}\right) .
\end{gathered}
$$

Next and on the other hand, by using the estimate expressed in one of the above previous equations, namely, $\Gamma_{k r}^{i}=F(\eta)\left(\eta^{\prime}\right)$, and its successive derivatives, together with the other ones previously determined, that is,

$$
\eta=O\left(\frac{h^{2}}{R^{2}}\right), \quad \eta^{\prime}=O\left(\frac{1}{R^{5 / 2}}\right), \quad \eta^{\prime \prime}=O\left(\frac{1}{R^{7 / 2}}\right), \ldots,
$$

we come to the conclusion that the same kind of estimates is valid for the corresponding covariant derivatives with respect to the Levi-Civita connection, the importance here being due to the fact that such kind of estimates is independent of the system of coordinates chosen. Thus, we state the main result of this section.

Theorem 6. With the above notations and conditions, there holds the following estimate:

$$
t_{k_{1}, k_{2} ; i_{1}, i_{2}, \ldots, i_{n}}=O\left(\varepsilon^{2} \lambda^{1-n} h^{-1}\right) .
$$

That is, in terms of a coordinate free expression,

$$
\left\|\widetilde{\nabla}^{n} t\right\|=O\left(\varepsilon^{2} \lambda^{1-n} h^{-1}\right) .
$$

\section{The Recovery of the Deformed Middle Surface}

The main objective in the theory of shells, whether Euclidean or affine, is to determine the extent of deformation of the shell 
once a solicitation is applied to it. And the best way of analyzing this is to see what happens to the corresponding middle surfaces. Thus, when the solicitation is produced on the shell in its original, undeformed state, it produces a threedimensional stress tensor [22-39]:

$$
t:=\sum_{i, j} t_{i j} d u^{i} d u^{j}=\sum_{i, j} t^{i j} \frac{\partial}{\partial u^{i}} \otimes \frac{\partial}{\partial u^{j}},
$$

which is well known to be symmetric; that is,

$$
t^{i j}=t^{j i}
$$

That first action produces, next, the deformation whose extent is contained as information in the corresponding strain tensor

$$
\varepsilon_{i j}:=\frac{1}{2}\left(G_{i j}^{*}-G_{i j}\right)
$$

Let us recall that the relation between both of them is established through the stress-strain relation as described in $[22,23]$ :

$$
t^{m k}:=\sqrt{\frac{G}{G^{*}}} \frac{\partial W}{\partial \varepsilon_{m k}} .
$$

This, in terms of tensors with mixed components, may also be written as

$$
t_{i}^{m}=\sqrt{\frac{G}{G^{*}}} \frac{\partial W}{\partial \varepsilon_{m}^{i}},
$$

as already analyzed, for the affine case, in our previous articles $[24,25]$. See also Section 5 above.

The earlier considerations, mainly the estimates exposed in the previous sections, together with the geometrical information available so far, shall be used in the recovery of the strained middle surface. We see, for example, from Section 2, that on the middle surface, characterized by the equation $u^{3}:=u=0$, we have

$$
G_{\alpha \beta}\left(u^{1}, u^{2}, 0\right)=g_{\alpha \beta}\left(u^{1}, u^{2}\right) .
$$

Hence and correspondingly, we may also write that

$$
G_{\alpha \beta}^{*}\left(u^{1}, u^{2}, 0\right):=g_{\alpha \beta}^{*}\left(u^{1}, u^{2}\right) .
$$

The main geometrical information is being contained in the middle surfaces $M_{0}$ and $M_{0}^{*}$ through the corresponding difference tensors $\varepsilon_{\alpha \beta}:=(1 / 2)\left(g_{\alpha \beta}^{*}-g_{\alpha \beta}\right), \sigma_{\alpha \beta \gamma}:=g_{\alpha \beta \gamma}^{*}-g_{\alpha \beta \gamma}$, and $w_{\alpha \lambda}:=B_{\alpha \lambda}^{*}-B_{\alpha \lambda}$, defined on the parameter space and relating the coefficients of the first, second, and third fundamental forms, treated in [24] and also exposed above.

Thus, the known data are those corresponding to the undeformed middle surface $M_{0}$, that is, $g_{\alpha \beta}, g_{\alpha \beta \gamma}$, and $B_{\alpha \lambda}$, and the problem is to determine the deformed ones, $g_{\alpha \beta}^{*}, g_{\alpha \beta \gamma}^{*}$, and $B_{\alpha \lambda}^{*}$, because these furnish a description of the deformed middle surface $M_{0}^{*}$, up to rigid motions in unimodular affine geometry. In case we want to obtain, that is, recover, the actual deformed middle surface we can do so by applying one, or more, of the several existence fundamental theorems available, as treated, for example, in [7] and, with different notations, in [12]. In these two books the cases of general dimensions are considered, while the version for surfaces is originally due to Radon [14]. The corresponding method for the recovery of the deformed middle surface had been previously treated, in the Euclidean case, by John in papers $[22,23]$, based on the well-known fundamental theorem for (Euclidean) surfaces as developed, for example, in [40-42].

The affine pseudo-Riemannian structure induced on the deformed shell may be represented by writing

$$
X^{*}\left(u_{1}, u_{2}, v\right)=X_{0}^{*}\left(u_{1}, u_{2}\right)+v N_{u a}^{*} .
$$

So, we may express the affine objects components, $g_{\alpha \beta}^{*}$, $g_{\alpha \beta \gamma}^{*}$, and $B_{\alpha \beta}^{*}$, which are going to play a role comparable to the ones in the Euclidean case, $a_{\alpha \beta}^{*}, L_{\alpha \beta}^{*}$, and $M_{\alpha \beta}^{*}$, developed previously by John in $[22,23]$. Here the treatment is made by assuming suitable integrability conditions exposed, for example, in [42] by Millman and Parker, where the fundamental theorem of existence and uniqueness (congruence) is stated, while complete proofs of the results can be found in [40] and also in [41]. On the other hand, the corresponding material for the affine case may be seen fully developed by Gigena, in [7], and, with a different kind of notation by Nomizu and Sasaki, in [12]. The expositions made in the latter two references are valid for dimension of the immersed hypersurface greater or equal than two, $n \geq 2$, while the original case of surfaces, that is, $n=2$, was treated by Radon in [14].

If we consider that the shell deformation is the same, whatever the geometry is, but treated in a different way for each case, we have the following relations.

Euclidean Gauss equation

$$
\left(X_{0}\right)_{\alpha \beta}^{*}=L_{\alpha \beta}^{*} N_{e u}^{*}+\Gamma_{\alpha \beta}^{* 1}\left(X_{0}\right)_{1}^{*}+\Gamma_{\alpha \beta}^{* 2}\left(X_{0}\right)_{2}^{*} \text {, }
$$

where, in the middle surface $(u=0)$, we have

$$
a_{\alpha \beta}^{*}=\left(X_{0}\right)_{1}^{*} \cdot\left(X_{0}\right)_{2}^{*}=X_{1}^{*}\left(u_{1}, u_{2}, 0\right) \cdot X_{2}^{*}\left(u_{1}, u_{2}, 0\right),
$$

affine Gauss equation

$$
\left(X_{0}\right)_{\alpha \beta}^{*}=g_{\alpha \beta}^{*} N_{u a}^{*}+\Gamma_{\alpha \beta}^{* 1}\left(X_{0}\right)_{1}^{*}+\Gamma_{\alpha \beta}^{* 2}\left(X_{0}\right)_{2}^{*} \text {. }
$$

Notice that the meaning for the coefficients $\Gamma_{\alpha \beta}^{* \lambda}$ is different in one or the other of the above equations. However, we have preferred to use the same kind of symbol in order to avoid unnecessary complications in notation.

Thus, by the very definition of the corresponding second fundamental (cubic) form

$$
\nabla\left(I_{u a}^{*}\right)=I I_{u a}^{*}
$$

we may write that

$$
g_{\alpha \beta \gamma}^{*}=g_{\alpha \beta ; \gamma}^{*}=\partial_{\gamma} g_{\alpha \beta}^{*}-\Gamma_{\alpha \gamma}^{* 1} g_{1 \beta}^{*}-\Gamma_{\beta \gamma}^{* 2} g_{2 \alpha}^{*} \text {. }
$$


It follows that we may successively write, by always reducing the situation to the middle surface $(u=0)$ at the end of the process,

$$
\begin{aligned}
\partial_{\beta} G_{\alpha s}^{*} & :=\frac{\partial}{\partial u^{\beta}} G^{*}\left(X_{\alpha}^{*}, X_{s}^{*}\right) \\
& =G^{*}\left(X_{\alpha \beta}^{*}, X_{s}^{*}\right)+G^{*}\left(X_{\alpha}^{*}, X_{s \beta}^{*}\right)
\end{aligned}
$$

and, by rotating indexes, we also have

$$
\begin{aligned}
\partial_{\alpha} G_{\beta s}^{*} & =\partial_{\alpha} G^{*}\left(X_{\beta}^{*}, X_{s}^{*}\right) \\
& =G^{*}\left(X_{\beta \alpha}^{*}, X_{s}^{*}\right)+G^{*}\left(X_{\beta}^{*}, X_{s \alpha}^{*}\right), \\
\partial_{s} G_{\alpha \beta}^{*} & =\partial_{s} G^{*}\left(X_{\alpha}^{*}, X_{\beta}^{*}\right) \\
& =G^{*}\left(X_{\alpha s}^{*}, X_{\beta}^{*}\right)+G^{*}\left(X_{\alpha}^{*}, X_{\beta s}^{*}\right) .
\end{aligned}
$$

Then, we readily get that

$$
\frac{1}{2}\left(\partial_{\beta} G_{\alpha s}^{*}+\partial_{\alpha} G_{\beta s}^{*}-\partial_{s} G_{\alpha \beta}^{*}\right)=G^{*}\left(X_{\alpha \beta}^{*}, X_{s}^{*}\right) .
$$

Now, from the latter we get successively that

$$
\begin{aligned}
\frac{1}{2} G^{* 3 s}\left(\partial_{\beta} G_{\alpha s}^{*}+\partial_{\alpha} G_{\beta s}^{*}-\partial_{s} G_{\alpha \beta}^{*}\right)=G^{*}\left(X_{\alpha \beta}^{*}, G^{* 3 s} X_{s}^{*}\right) \\
G^{*}\left(X_{\alpha \beta}^{*}, G^{* 3 s} X_{s}^{*}\right) \\
=G^{* 33} g_{\alpha \beta}^{*} G^{*}\left(N_{u a}^{*}, X_{3}^{*}\right) \\
+\Gamma_{\alpha \beta}^{* 1}\left(G^{* 31} G_{11}^{*}+G^{* 32} G_{21}^{*}+G^{* 33} G_{31}^{*}\right) \\
+\Gamma_{\alpha \beta}^{* 2}\left(G^{* 21} G_{12}^{*}+G^{* 22} G_{22}^{*}+G^{* 23} G_{32}^{*}\right)
\end{aligned}
$$

where we further observe that the last two terms vanish since

$$
\begin{aligned}
& G^{* 3 s} G_{s 1}^{*}=\delta_{1}^{3}=0, \\
& G^{* 3 s} G_{s 2}^{*}=\delta_{2}^{3}=0 .
\end{aligned}
$$

On the other hand we may also write similarly

$$
\begin{aligned}
G^{*}\left(X_{\alpha \beta}^{*}, G^{* 2 s} X_{s}^{*}\right)= & G^{* 23} g_{\alpha \beta}^{*} G^{*}\left(N_{u a}^{*}, X_{3}^{*}\right) \\
& +\Gamma_{\alpha \beta}^{* 1} \underbrace{\left(G^{* 21} G_{11}^{*}+G^{* 22} G_{21}^{*}+G^{* 23} G_{31}^{*}\right)}_{\delta_{1}^{2}=0} \\
& +\Gamma_{\alpha \beta}^{* 2} \underbrace{\left(G^{* 21} G_{12}^{*}+G^{* 22} G_{22}^{*}+G^{* 23} G_{32}^{*}\right)}_{\delta_{2}^{2}=1}, \\
G^{*}\left(X_{\alpha \beta}^{*}, G^{* 1 s} X_{s}^{*}\right)= & G^{* 13} g_{\alpha \beta}^{*} G^{*}\left(N_{u a}^{*}, X_{3}^{*}\right) \\
& +\Gamma_{\alpha \beta}^{* 1} \underbrace{\left(G^{* 11} G_{11}^{*}+G^{* 12} G_{21}^{*}+G^{* 13} G_{31}^{*}\right)}_{\delta_{1}^{1}=1} \\
& +\Gamma_{\alpha \beta}^{* 2} \underbrace{\left(G^{* 11} G_{12}^{*}+G^{* 12} G_{22}^{*}+G^{* 13} G_{32}^{*}\right)}_{\delta_{2}^{1}=0} .
\end{aligned}
$$

Now, in order to properly express the term $G^{*}\left(N_{u a}^{*}, X_{3}^{*}\right)$, appearing in all of these equations, we may proceed to use the very well-known Gram determinant identities:

$$
\begin{aligned}
& {\left[X_{1}^{*}, X_{2}^{*}, N_{u a}^{*}\right]\left[X_{1}^{*}, X_{2}^{*}, X_{3}^{*}\right]} \\
& \quad= \pm \operatorname{det}\left[\begin{array}{ccc}
G_{11}^{*} & G_{12}^{*} & G_{13}^{*} \\
G_{12}^{*} & G_{22}^{*} & G_{23}^{*} \\
0 & 0 & G^{*}\left(N_{u a}^{*}, X_{3}^{*}\right)
\end{array}\right],
\end{aligned}
$$

so that

$$
\left[X_{1}^{*}, X_{2}^{*}, N_{u a}^{*}\right]\left[X_{1}^{*}, X_{2}^{*}, X_{3}^{*}\right]= \pm G^{*}\left(N_{u a}^{*}, X_{3}^{*}\right) \operatorname{det}\left[G_{\alpha \beta}^{*}\right] .
$$

Following the same reasoning, we also have

$$
\begin{gathered}
{\left[X_{1}^{*}, X_{2}^{*}, X_{3}^{*}\right]^{2}= \pm \operatorname{det}\left[G_{i j}^{*}\right],} \\
{\left[X_{1}^{*}, X_{2}^{*}, N_{u a}^{*}\right]^{2}= \pm \operatorname{det}\left[\begin{array}{ccc}
G_{11}^{*} & G_{12}^{*} & 0 \\
G_{12}^{*} & G_{22}^{*} & 0 \\
0 & 0 & 1
\end{array}\right]= \pm \operatorname{det}\left[\begin{array}{ll}
G_{11}^{*} & G_{12}^{*} \\
G_{12}^{*} & G_{22}^{*}
\end{array}\right] .}
\end{gathered}
$$

Thus, we obtain

$$
G^{*}\left(N_{u a}^{*}, X_{3}^{*}\right)=\frac{\left[X_{1}^{*}, X_{2}^{*}, X_{3}^{*}\right]}{\left[X_{1}^{*}, X_{2}^{*}, N_{u a}^{*}\right]}=\sqrt{\frac{\operatorname{det}\left[G_{i j}^{*}\right]}{\operatorname{det}\left[G_{\alpha \beta}^{*}\right]}}=\left(G^{* 33}\right)^{-1 / 2} .
$$

So far, from the above it follows that the coefficients of the affine Gauss equation may be expressed in terms of the components of the deformed pseudometric of the shell. In fact, see the expressions above containing the components $g_{\alpha \beta}^{*}, \Gamma_{\alpha \beta}^{* 1}$, and $\Gamma_{\alpha \beta}^{* 2}$.

It then follows that the rest of components of the first, second, and third affine fundamental forms may be expressed, too, in terms of the components of the deformed pseudometric shell $G_{i j}^{*}$, in every case reducing those terms to the middle surface $(u=0)$.

From the information recorded in this way, we can properly approximate the first, second, and third fundamental forms of affine geometry and, by the further use of the Fundamental Existence Theorem, recover the deformed middle surface. See $[7,12,14]$, where the first two expositions are valid for the cases of the dimension of the immersed manifold $n \geq 2$, while the third one is valid for $n=2$.

Theorem 7. One may represent the deformed middle surface $M_{0}^{*}$ as a real, 2-dimensional, oriented differentiable manifold, together with the following geometrical objects: a torsion-free affine connection $\nabla^{*}$, a nondegenerate, pseudo-Riemannian structure, $g^{*}$, and $a(1,1)$-tensor field, $S^{*}$, all of them defined in terms of the known, estimated coefficients of the deformed pseudometric exposed above, in Section 5:

$$
\begin{aligned}
G_{i k}^{*}= & G_{i k}+\frac{2}{\mu} t_{i k}-2\left(\frac{1-2 \mu}{2 \mu}\right)\left(\sum_{j} t_{j}^{j}\right) \delta_{i k} \\
& +F(t, \eta)\left(t^{2}+\eta t\right) .
\end{aligned}
$$


In fact, those geometrical objects satisfy the following integrability conditions.

(1) $g^{*}$ is a Codazzi tensor field with respect to $\nabla^{*}$, that is, in regard to the connection $\nabla^{*}$, the covariant derivative of $g^{*}$ is a symmetric $(0,3)$-tensor field, so that we can write

$$
\psi^{*}:=\nabla^{*} g^{*}=g_{\alpha \beta \gamma}^{*} d u^{\alpha} d u^{\beta} d u^{\gamma},
$$

with the local components $g_{\alpha \beta \gamma}^{*}$ symmetric in all of their indices.

(2) $\psi^{*}=\nabla^{*} g^{*}$ is apolar with respect to $g^{*}$; namely, the Tschebyscheff covector field vanishes identically, or in local coordinates $\tau^{*}:=\sum g^{* \alpha \beta} g_{\alpha \beta \gamma}^{*} d u^{\gamma} \equiv 0$.

(3) $S^{*}$ is a $(1,1)$-Codazzi tensor field with respect to $\nabla^{*}$; that is, the equation following holds:

$$
B_{\beta ; \gamma}^{* \alpha}=B_{\gamma ; \beta}^{* \alpha} \text {. }
$$

(4) The equation of Gauss holds, relating the given data, that is,

$$
\begin{aligned}
\widetilde{R}_{\beta \gamma \eta}^{* \alpha}= & \frac{1}{2}\left(B_{\eta}^{* \alpha} g_{\beta \gamma}^{*}-B_{\gamma}^{* \alpha} g_{\beta \eta}^{*}+B_{\beta \gamma}^{*} \delta_{\eta}^{\alpha}-B_{\beta \eta}^{*} \delta_{\gamma}^{\alpha}\right) \\
& -\sum\left(A_{\beta \eta}^{* \sigma} A_{\sigma \gamma}^{* \alpha}-A_{\beta \gamma}^{* \sigma} A_{\sigma \eta}^{* \alpha}\right),
\end{aligned}
$$

where $\widetilde{R}_{\beta \gamma \eta}^{* \alpha}$ represent the components of the curvature tensor associated with the Levi-Civita connection and $A_{\beta \gamma}^{* \alpha}$ are the components of the difference tensor, also equal to $(1 / 2) g^{* \alpha \rho} g_{\rho \beta \gamma}^{*}$.

Finally and consequently, it also follows that there exists a local unimodular affine immersion $X^{*}:\left(M^{*}, \nabla^{*}, g^{*}, S^{*}\right) \rightarrow$ $\mathbb{R}^{3}$ for which $\nabla^{*}$ is the affine normal connection, $g^{*}$ is the first fundamental form $I_{u a}^{*}$, and $S^{*}$ is Weingarten's affine shape operator.

Proof. The integrability conditions stated are a direct consequence of the corresponding compatibility conditions, as exposed in Theorem 4 above, and the rest of the proof, in a far more general setting and for general dimension $n \geq 2$, may be found in [7] and also in [12], the latter with a different kind of notation. The original case of dimension $n=2$ may be found in [14].

Moreover, for dimension $n=2$, we may further reduce the proof to a two-step procedure, whereas we consider first the expression involving the affine Gauss equation, together with the expression of the local derivatives of the affine normal vector field:

$$
\begin{aligned}
& \left(X_{0}\right)_{\alpha \beta}^{*}=g_{\alpha \beta}^{*} N_{u a}^{*}+\Gamma_{\alpha \beta}^{* 1}\left(X_{0}\right)_{1}^{*}+\Gamma_{\alpha \beta}^{* 2}\left(X_{0}\right)_{2}^{*}, \\
& \left(N_{u a}^{*}\right)_{\alpha}=-\sum_{\beta} B_{\alpha}^{* \beta}\left(X_{0}\right)_{\beta}^{*} .
\end{aligned}
$$

This is a system of five partial differential equations, when considering that it involves the vector fields $\left(X_{0}\right)_{11}^{*}$,
$\left(X_{0}\right)_{12}^{*},\left(X_{0}\right)_{22}^{*},\left(N_{u a}^{*}\right)_{1}$, and $\left(N_{u a}^{*}\right)_{2}$. Correspondingly, we have a system of fifteen scalar partial differential equations, when expressing each one of the latter in terms of the ambient space coordinates. The fact that the system is integrable, and allows recovering firstly the vector fields $\left(X_{0}\right)_{1}^{*},\left(X_{0}\right)_{2}^{*}$, and $N_{u a}^{*}$ follows precisely from the integrability conditions exposed, as stated before and, for example, in [7] and also in [12], the latter with different notation.

Thus, with this first step of the procedure we obtain those three vector fields in terms of the components of the deformed pseudometric shell $G_{i j}^{*}$, having always in mind their approximate values and also by considering the reduction of those same values to the deformed middle surface $(u=0)$.

In the next, second step of the procedure, we use those expressions for $\left(X_{0}\right)_{1}^{*}$ and $\left(X_{0}\right)_{2}^{*}$ in order to recover the vector field representing the deformed middle surface $\left(X_{0}\right)^{*}$. Here the integrability conditions are obviously satisfied since $\left(X_{0}\right)_{12}^{*}=\left(X_{0}\right)_{21}^{*}$.

\section{Conflict of Interests}

The authors declare that there is no conflict of interests regarding the publication of this paper.

\section{References}

[1] W. Blaschke, Vorlesungen Über Differentialgeometrie II, Springer, 1923.

[2] E. Calabi, "Improper Affine Hyperspheres of Convex Type and a Generalization of a Theorem by K. Jörgens," Michigan Mathematical Journal, vol. 5, pp. 105-126, 1958.

[3] E. Calabi, "Complete affine hyperspheres I.," in Symposia Mathematica, vol. 10, pp. 19-38, Istituto Nazionale di Alta Matematica, Academic Press, 1972.

[4] E. Calabi, "Hypersurfaces with maximal affinely invariant area," American Journal of Mathematics, vol. 104, no. 1, pp. 91-126, 1982.

[5] S. Y. Cheng and S. T. Yau, "Complete affine hypersurfaces. I: the completeness of affine metrics," Communications in Pure and Applied Mathematics, vol. 39, no. 6, pp. 839-866, 1986.

[6] S. S. Chern, Affine Minimal Hypersurfaces, Proceedings JapanU.S. Seminar on Minimal Submanifolds and Geodesics, Kaigai Publications, Tokyo, 1978.

[7] S. Gigena, Hypersurface Geometry And Related Invariants in A Real Vector Space, Research Monograph, Editorial Ingreso, Córdoba, Argentina, 1996.

[8] S. Gigena, "Classificatory problems in affine geometry approached by differential equations methods," Revista De La Unión Matemática Argentina, vol. 47, no. 2, pp. 95-107, 2007.

[9] E. Gläsner, "Ein affinanalogon zu den Scherksen minimalflächen," Archiv der Mathematik, vol. 27, pp. 436-439, 1977.

[10] A.-M. Li, "Calabi conjecture on hyperbolic affine hyperspheres," Mathematische Zeitschrift, vol. 203, no. 1, pp. 483-491, 1990.

[11] K. Nomizu and U. Pinkall, "Cubic form theorem for affine immersions," Results in Mathematics, vol. 13, no. 3-4, pp. 338362, 1988.

[12] K. Nomizu and T. Sasaki, Affine Differential Geometry, Cambridge University Press, 1994.

[13] A. V. Pogorelov, "On the improper convex affine hyperspheres," Geometriae Dedicata, vol. 1, no. 1, pp. 33-46, 1972. 
[14] J. Radon, "Die grundgleichungen der affinen flächentheorie," Leipziger Berichte, vol. 70, pp. 91-107, 1918.

[15] T. Sasaki, "Hyperbolic affine hyperspheres," Nagoya Mathematical Journal, vol. 77, pp. 107-123, 1980.

[16] P. A. Schirokov and A. P. Schirokov, Affine Differentialgeometrie, Teubner, Leibzig, Germany, 1962.

[17] R. Schneider, "Zur affinen Differentialgeometrie im Großen. I," Mathematische Zeitschrift, vol. 101, no. 5, pp. 375-406, 1967.

[18] R. Schneider, "Zur affinen Differentialgeometrie im Großen II. Über eine Abschätzung der Pickschen Invariante auf Affinsphären," Mathematische Zeitschrift, vol. 102, no. 1, pp. 1-8, 1967.

[19] G. Tzitzeica, "Sur une nouvelle classe des surfaces," Rendiconti del Circolo Matematico di Palermo, vol. 25, no. 1, pp. 180-187, 1908.

[20] G. Tzitzeica, "Sur une nouvelle classe des surfaces," Rendiconti del Circolo Matematico di Palermo, vol. 28, no. 1, pp. 210-216, 1909.

[21] L. Vrancken, "Affine higher order parallel hypersurfaces," Annales de la Faculté des Sciences de Toulouse, vol. 9, no. 3, pp. 341353, 1988.

[22] F. John, "Estimates for the derivatives of the stresses in a thin shell and interior shell equations," Communications on Pure and Applied Mathematics, vol. 18, no. 1-2, pp. 235-267, 1965.

[23] F. John, "Refined interior equations for thin elastic shells," Communications on Pure and Applied Mathematics, vol. 24, no. 5, pp. 583-615, 1971.

[24] S. Gigena, M. Binia, and D. Abud, "Condiciones de compatibilidad para cáscaras afines," Mecánica Computacional, vol. 21, pp. 1862-1881, 2002.

[25] S. Gigena, D. Abud, and M. Binia, "Theory of affine shells: higher order estimates," Mecánica Computacional, vol. 29, pp. 969988, 2010.

[26] R. A. Arciniega and J. N. Reddy, “Tensor-based finite element formulation for geometrically nonlinear analysis of shell structures," Computer Methods in Applied Mechanics and Engineering, vol. 196, no. 4-6, pp. 1048-1073, 2007.

[27] A. E. Green and W. Zerna, Theoretical Elasticity, Oxford University Press, 1954.

[28] S. Klinkel, F. Gruttmann, and W. Wagner, "Continuum based three-dimensional shell element for laminated structures," Computers and Structures, vol. 71, no. 1, pp. 43-62, 1999.

[29] W. T. Koiter, "A systematic simplification of the general equations in the linear theory of thin shells," Nederlandse Akademie van Wetenschappen, vol. 42, pp. 263-277, 1964.

[30] W. T. Koiter, "A comparison between John's refined interior shell equations and classical shell theory," Zeitschrift für Angewandte Mathematik und Physik ZAMP, vol. 20, no. 5, pp. 642-652, 1969.

[31] W. T. Koiter, "On the mathematical foundation of shell theory," Proceedings International Congress of Mathematicians, vol. 3, pp. 123-130, 1970.

[32] W. T. Koiter and J. C. Simmonds, "Foundations of shell theory," in Proceedings of the 13th International Congress of Theoretical and Applied Mechanics, pp. 150-176, 1973.

[33] V. Lods and C. Mardare, "A justification of linear Koiter and Naghdi's models for totally clamped shell," Asymptotic Analysis, vol. 31, no. 3-4, pp. 189-210, 2002.

[34] A. E. H. Love, A Treatise on the Mathematical theory of Elasticity, Dover, 4th edition, 1944.

[35] R. H. Macneal and R. L. Harder, "A proposed standard set of problems to test finite element accuracy," Finite Elements in Analysis and Design, vol. 1, no. 1, pp. 3-20, 1985.
[36] H. Möllmann, Introduction To the Theory of Thin Shells, John Wiley \& Sons, 1981.

[37] J. N. Reddy, An Introduction To Continuum Mechanics, Cambridge University Press, 2008.

[38] E. Reissner, "On the derivation of the theory of thin elastic shells," Journal of Mathematical Physics, vol. 42, pp. 263-277, 1964.

[39] W. Wagner and F. Gruttmann, "A simple finite rotation formulation for composite shell elements," Engineering Computations, vol. 11, no. 2, pp. 145-176, 1994.

[40] C. C. Hsiung, A First Course in Differential Geometry, John Wiley \& Sons, 1981.

[41] W. Klingenberg, A Course in Differential Geometry, Springer, 1978.

[42] R. Millman and G. Parker, Elements of Differential Geometry, Prentice-Hall, New Jersey, NJ, USA, 1977. 


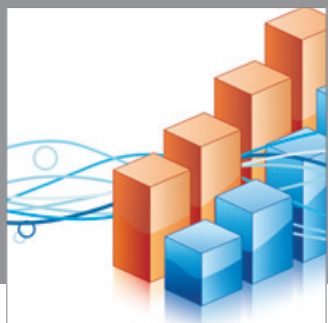

Advances in

Operations Research

mansans

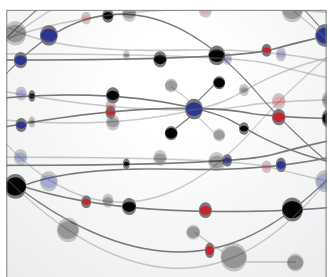

The Scientific World Journal
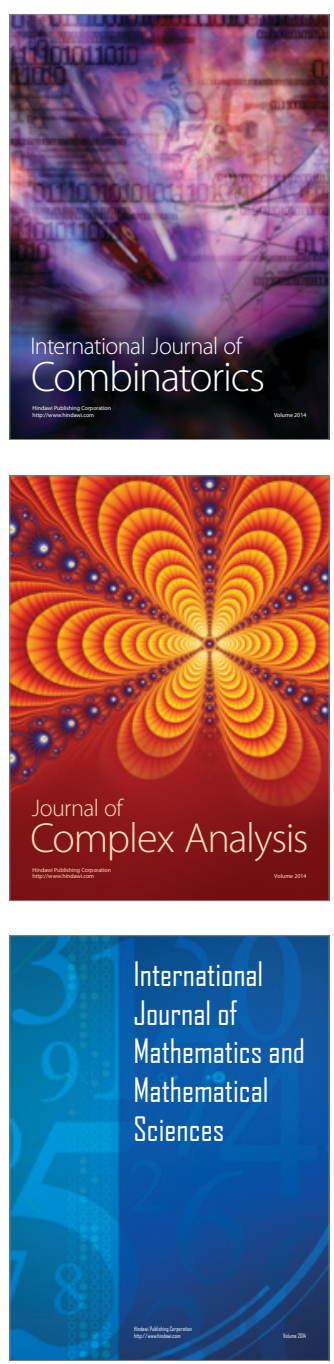
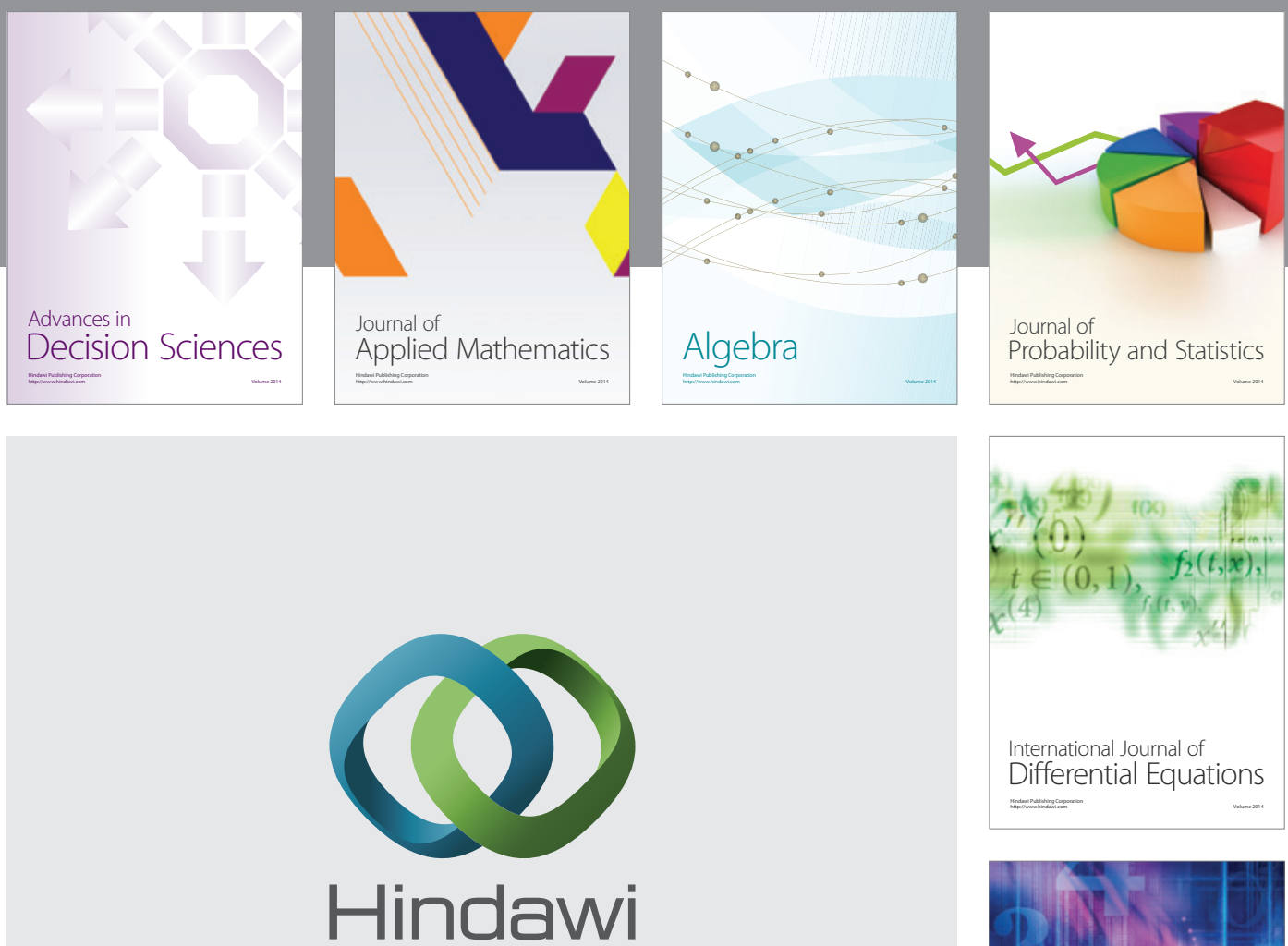

Submit your manuscripts at http://www.hindawi.com
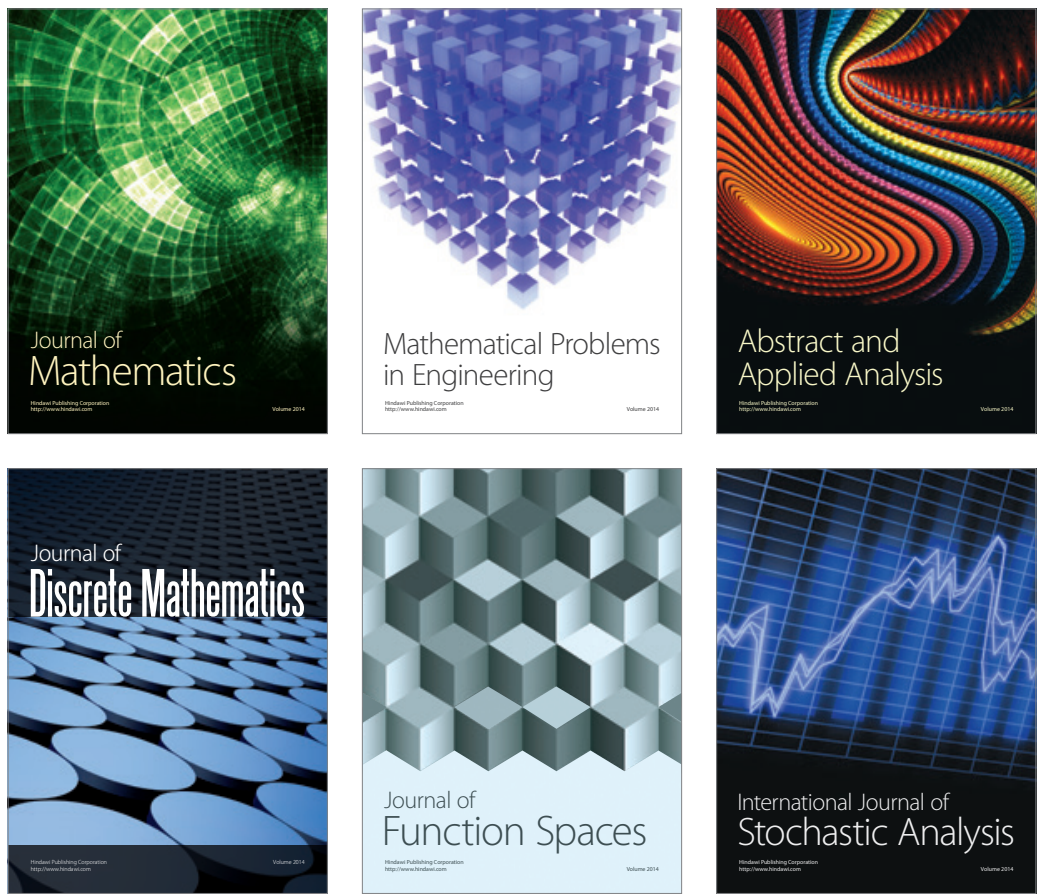

Journal of

Function Spaces

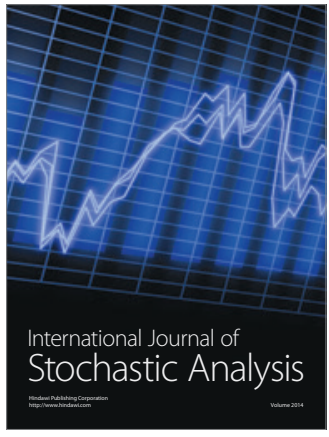

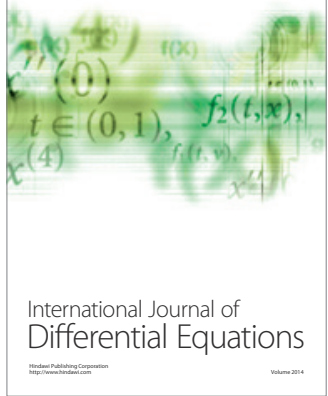
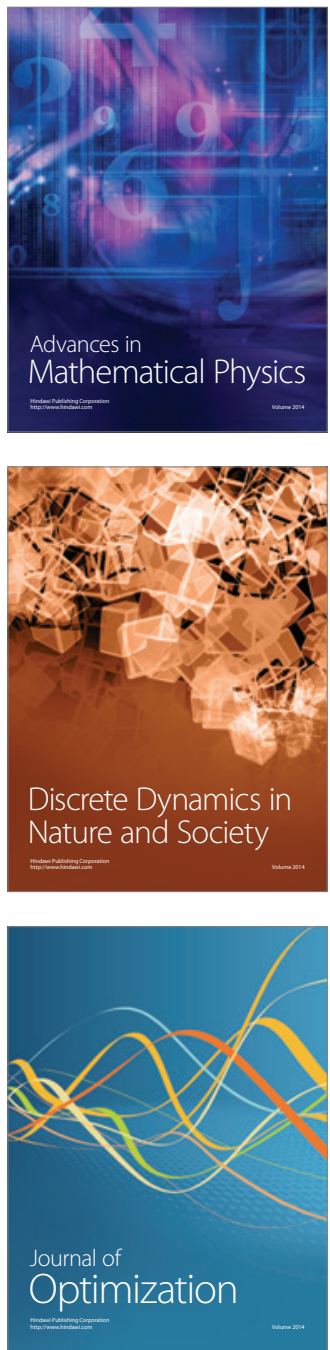